\title{
Ladder Treadmill: A Method to Assess Locomotion in Cats with an Intact or Lesioned Spinal Cord
}

\author{
Manuel Escalona, Hugo Delivet-Mongrain, Aritra Kundu, Jean-Pierre Gossard, and Serge Rossignol \\ Groupe de Recherche sur le Système Nerveux Central, Department of Neurosciences, Université de Montréal, Montreal, Quebec H3T 1J4, Canada
}

After lesions of the CNS, locomotor abilities of animals (mainly cats) are often assessed on a simple flat treadmill (FTM), which imposes little demands on supraspinal structures as is the case when walking on targets. Therefore, the aims of the present work were as follows: (1) to develop a treadmill allowing the assessment of locomotion of intact cats required to place the paws on the rungs of a moving ladder treadmill (LTM); (2) to assess the capability of cats after a unilateral spinal hemisection at T10 to cope with such a demanding locomotor task; and (3) to regularly train cats for 6 weeks on the LTM to determine whether such regular training improves locomotor recovery on the FTM. A significant improvement would indicate that LTM training maximizes the contribution of spinal locomotor circuits as well as remnant supraspinal inputs. Together, we used 9 cats ( 7 females, 2 males). Six were used to compare the EMG and kinematic locomotor characteristics during walking on the FTM and LTM. We found that the swing phase during LTM walking was slightly enhanced as well as some specific activity of knee flexor muscles. Fore-hindlimb coupling favored a more stable diagonal coupling. These 6 cats were then hemispinalized and trained for 6 weeks on the LTM, whereas the 3 other cats were hemispinalized and trained solely on the FTM to compare the two training regimens. Intensive LTM training after hemisection was found to change features of locomotion, such as the foot trajectory as well as diminished paw drag often observed after hemisection.

Key words: electromyography; kinematics; ladder treadmill; locomotion; locomotor training; spinal hemisection

Significance Statement

This paper introduces a method (ladder treadmill [LTM]) to study the locomotor ability of cats with an intact spinal cord or after a unilateral hemisection to walk with a precise foot placement on the rungs fixed to an ordinary flat treadmill (FTM). Because cats are compared in various conditions (intact or hemisected at different time points) in the same enclosure on the FTM and the LTM, the changes in averaged locomotor characteristics must reflect the specificity of the task and the neurological states. Furthermore, the ladder treadmill permits to train cats repetitively for weeks and observe whether training regimens (FTM or LTM) can induce durable changes in the parameters of locomotion.

\section{Introduction}

Locomotor training on a treadmill or a walkway after spinal cord injury has become a widespread rehabilitation approach in animal models (Edgerton et al., 2001; Alluin et al., 2014; Rossignol et al., 2015) as well as in humans (Dietz, 1995; Harkema, 2001; Barbeau et al., 2002; Field-Fote et al., 2005; Harkema et al., 2011;

Received Jan. 5, 2017; revised April 18, 2017; accepted April 25, 2017.

Author contributions: J.-P.G. and S.R. designed research; M.E., H.D.-M., and A.K. performed research; M.E. and H.D.-M. analyzed data; J.-P.G. and S.R. wrote the paper.

This work was supported by Wings For Life Contract WFL-CA-030-14 and Canadian Institutes of Health Research Grant to J.-P.G. and Emerging Team Grant (SMRRT) and Canada Research Chair to S.R. M.E. and A.K. were supported by Win For Life studentships. All studies were conducted in the Department of Neurosciences, Faculty of Medicine the Université de Montréal, Montreal, Quebec H3T 1J4, Canada.

The authors declare no competing financial interests.

Correspondence should be addressed to Dr. Serge Rossignol, Groupe de Recherche sur le Système Nerveux Central, Faculté de Médecine, Université de Montréal, Department of Neurosciences, Pavillon Paul-G.-Desmarais, 2960 Chemin de la Tour, Montreal, Quebec H3T 1J4, Canada. E-mail: serge.rossignol@umontreal.ca.

DOI:10.1523/JNEUROSCI.0038-17.2017

Copyright $\odot 2017$ the authors $\quad 0270-6474 / 17 / 375429-18 \$ 15.00 / 0$
A. C. Smith et al., 2014). As reviewed previously (Edgerton et al., 2001; Rossignol et al., 2015), among the benefits of such training, in which step cycles are repeated for several minutes each day for several consecutive weeks, are the increase of muscle force output (Hodgson et al., 1994) and endurance (De Leon et al., 1999) as well as a strengthening of spinal locomotor circuitry whose rhythmic activity can be recorded even after paralysis during fictive locomotion without drug stimulation (Pearson and Rossignol, 1991). Long-term locomotor training can progressively influence the evolution and quality of the locomotor pattern in cats (Barbeau and Rossignol, 1987). Furthermore, it is possible to train specific motor patterns (e.g., walking vs standing) (Edgerton et al., 1997; De Leon et al., 1998). In the case of spinal animals, repetition of the same movements may, through the activation of movementrelated afferent feedback, induce the necessary plastic modifications of intrinsic spinal locomotor circuits.

After partial spinal cord lesions, treadmill training also affects the characteristics and quality of walking through more complex 
mechanisms because not only spinal circuits but also remnant descending/ascending pathways may participate in controlling locomotor recovery. Locomotor training is usually limited to repetitive simple locomotor movements on a flat treadmill (FTM). In our own studies of cats after a unilateral hemisection, recovery of quadrupedal locomotion was remarkable. Some minor remaining deficits, such as foot drag and asymmetrical hindlimb coupling, could largely be corrected with daily locomotor training on the ordinary FTM (Barrière et al., 2008, 2010; Martinez et al., 2011). However, we did not investigate systematically the capacity of these animals to walk voluntarily on specific targets or avoid obstacles that require supraspinal visual inputs. Work in cats (Lavoie et al., 1995; Drew et al., 2008; Farrell et al., 2015) and humans (Patla et al., 1991) has shown the critical importance of direct or indirect cortical mechanisms in controlling precision walking; these mechanisms could participate in the recovery of hemisected cats. We thus developed a repetitive locomotor task that requires some precision walking as previously shown by previous work in a different context and for different needs (Amos et al., 1987; Armstrong and Marple-Horvat, 1996).

We designed a ladder treadmill (LTM) to assess locomotion in cats that could be trained for several minutes every day in a condition requiring a repetitive accurate placement of all feet on rungs fixed to our ordinary FTM belt. By removing the rungs, we could also assess and compare locomotor characteristics (kinematics, electromyography) on the LTM and FTM within the same enclosure and in the same experimental recording session on any given day. Furthermore, cats could be filmed and recorded continuously at a fixed position relative to the treadmill enclosure, allowing averaging of data over 14-20 steps at the same location in each condition. After establishing the baseline values comparing FTM and LTM walking in a group of control cats, 9 cats were further submitted to a unilateral spinal hemisection at T10. Three of the 9 cats were daily trained on the FTM exclusively, whereas the 6 other cats were trained on the LTM for up to $42 \mathrm{~d}$. Some task-specific electromyographic and kinematics changes were observed, suggesting that some task-dependent locomotor features can be long lasting.

It is thought that comparing locomotion and its recovery on an LTM and or FTM will improve our understanding of normal locomotor control as well as better characterize impairments after specific lesions of the CNS. The possibility of averaging several consecutive step cycles in the same environment while imposing various locomotor demands should facilitate such quantitative comparisons.

\section{Materials and Methods}

Animal care. All procedures followed a protocol approved by the Ethics Committee at the Universite de Montréal, according to the Canadian guide for the care and use of experimental animals. The well-being of the cats was monitored daily and verified regularly by a veterinarian. After EMG implantation and spinal lesions (see below), cats were housed in individual cages $(104 \times 76 \times 94 \mathrm{~cm})$ with food and water ad libitum. Such housing limits self-training outside the locomotor training sessions.

LTM. Quadrangular hard foam rungs $(35 \mathrm{~cm}$ length, $5 \mathrm{~cm}$ width, and $5 \mathrm{~cm}$ height) were attached perpendicularly to the treadmill belt on which Velcro bands were glued on each side. Rungs were spaced 6-8 cm apart to match the most regular sequences of step length (i.e., the spacing that allowed a given cat to consistently place the paws at every two rungs; see Fig. 1A).

Experimental paradigm. Adult cats ( $n=9 ; 2$ males, 7 females), weighing 3.3-4 kg, were first selected for their ability to walk regularly for several minutes $(15-30 \mathrm{~min})$ on the FTM and on the LTM. Figure $1 B$ illustrates the sequence of events of the training paradigm. EMG elec- trodes were implanted in representative flexor and extensor muscles of the forelimbs and hindlimbs bilaterally. After EMG implantation, two testing sessions were made to verify the quality of the EMG signals and locomotor movements. Then, kinematic and EMG values in the two conditions (FTM or LTM) were recorded on the same day for each cat and at the same speed $(0.5 \mathrm{~m} / \mathrm{s})$. After collecting baseline values on the FTM, a left hemisection was performed at thoracic 10 (T10 vertebra). For the next 6 weeks, 6 cats were trained on the LTM and the 3 others on the FTM, 5 times a week for $30 \mathrm{~min}$. Once a week, the stepping patterns (EMG, kinematics) were recorded together with synchronized videos at $0.5 \mathrm{~m} / \mathrm{s}$ on the FTM and on the LTM. The outcome, the stepping performance on the FTM, was recorded, analyzed, and compared for both groups of cats. Significant differences between the two groups were taken as evidence for specific training-related plasticity.

Spinal lesions. The general procedure for spinal lesions was similar to that described in our previous studies (Barrière et al., 2008; Martinez et al., 2011). T10-T11 vertebrae were exposed, and a small laminectomy was performed to approach the spinal cord dorsally. A small incision of the dura was first made, and a few drops of a local anesthetic ( $2 \%$ xylocaine) were injected on the top of, and directly into, the targeted segment to reduce the shock of sectioning. Hemisections on the left side were achieved with micro-scissors under a microscope. The wound was then closed in anatomic layers.

Surgical procedures. All surgical procedures for electrode implantation were done under general anesthesia and aseptic conditions as described previously (Martinez et al., 2013). Briefly, animals were first premedicated with Atravet $(0.1 \mathrm{mg} / \mathrm{kg})$, glycopyrrolate $(0.01 \mathrm{mg} / \mathrm{kg})$, and ketamine $(10 \mathrm{mg} / \mathrm{kg})$. An endotracheal tube was then inserted for gaseous anesthesia (mixture of $\mathrm{O}_{2}$ and isoflurane 2\%).

EMG implantations. Cats were chronically implanted with intramuscular electrodes to record EMG activity from flexor and extensor muscles in the hindlimb and forelimb on both sides. The implanted muscles were semitendinosus (knee flexor and hip extensor), sartorius (hip flexion and knee extension), vastus lateralis (VL; knee extensor), gastrocnemius medialis (GM; ankle extensor and knee flexor), tibialis anterior (ankle flexor), extensor digitorum brevis (EDB; dorsiflexor of the hindpaw digits), triceps brachii (elbow extensor), and biceps brachii (elbow flexor). Electrodes were led subcutaneously to two 15-pinhead connectors secured to the cranium using acrylic cement. Heart rate and respiration were monitored throughout the surgeries. At $24 \mathrm{~h}$ before surgery, an antibiotic (Convenia, $8 \mathrm{mg} / \mathrm{kg}$ ) was administered subcutaneously. Before the end of surgery, an analgesic (buprenorphine, $0.01 \mathrm{mg} / \mathrm{kg}$ ) was administered subcutaneously. Additionally, a fentanyl patch $(25 \mu \mathrm{g} / \mathrm{h})$ was sutured to the skin to alleviate pain for $\sim 5 \mathrm{~d}$.

Kinematic and EMG recordings. During episodes of locomotion, the stepping patterns of cats were recorded on video from the left side with a digital camera and the data stored on a hard disk. Video images $(30 \mathrm{~Hz})$ were deinterlaced to yield a resolution of 60 fields/s or $16.6 \mathrm{~ms}$ between fields. Reflective markers were placed over the iliac crest, greater trochanter, lateral malleolus, metatarsophalangeal (MTP) joint, and one at the tip of the toes of the left hindlimb (LH) (see Fig. 1A). This last marker was used to visually tag foot contact and lift off on video images and determine the swing and stance phases. It also allowed us to study the left paw trajectory during the swing phase. For the forelimbs, manually placed reflective markers were also used to identify foot contacts and lift off.

The amplified (Lynx-8 amplifiers, Neuralynx) and filtered (bandwidth $100 \mathrm{~Hz}$ to $3 \mathrm{kHz}$ ) EMG signals were digitized at $1 \mathrm{kHz}$ (NI-6071E, National Instruments) and stored in a computer. Kinematic and EMG recordings were synchronized using a Society of Motion Picture and Television Engineers time code generator.

Kinematics and EMG analyses. Step cycle duration. Step cycle duration represents the time between two successive contacts of the same foot on the treadmill, whereas the stance duration refers to the time between foot contact and toe off, which corresponds to the initiation of the swing phase of a single limb. To avoid bias from subtle changes in the walking speed or unintentional displacements of the camera, the $x$ coordinates of the toe point were normalized to the hip marker fixed to the iliac crest. This ensured that all angular measurements were referred to the same point in space in the $x$-axis. 
A

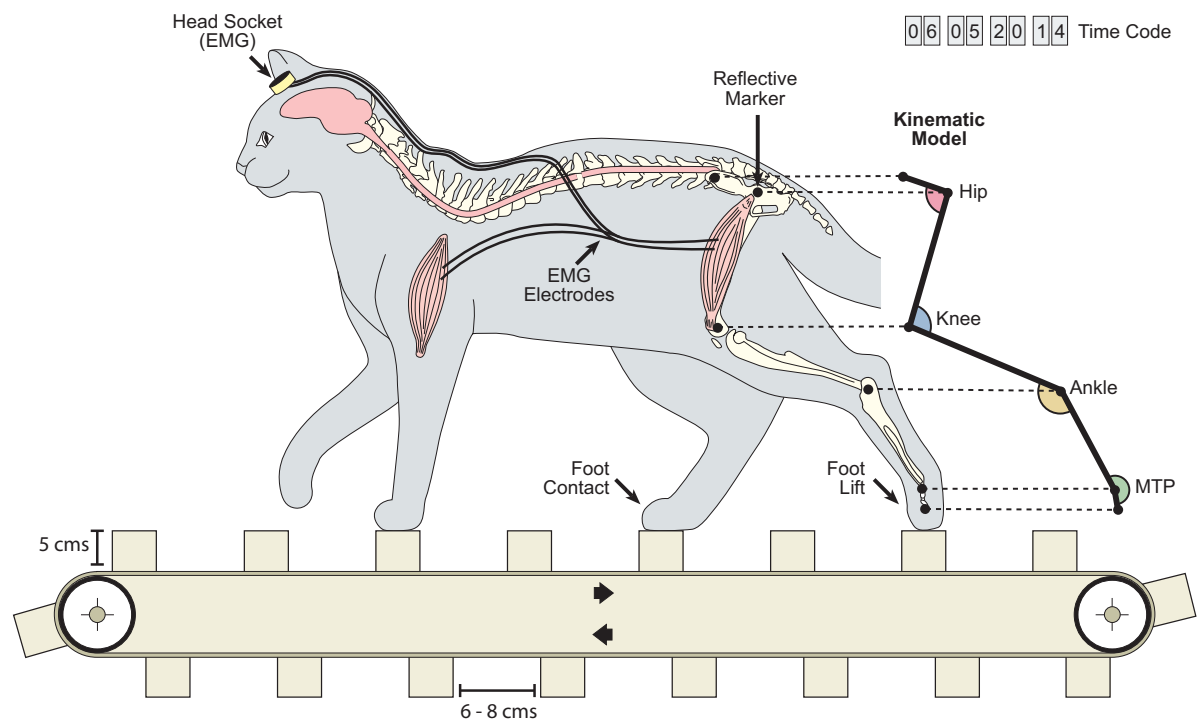

B

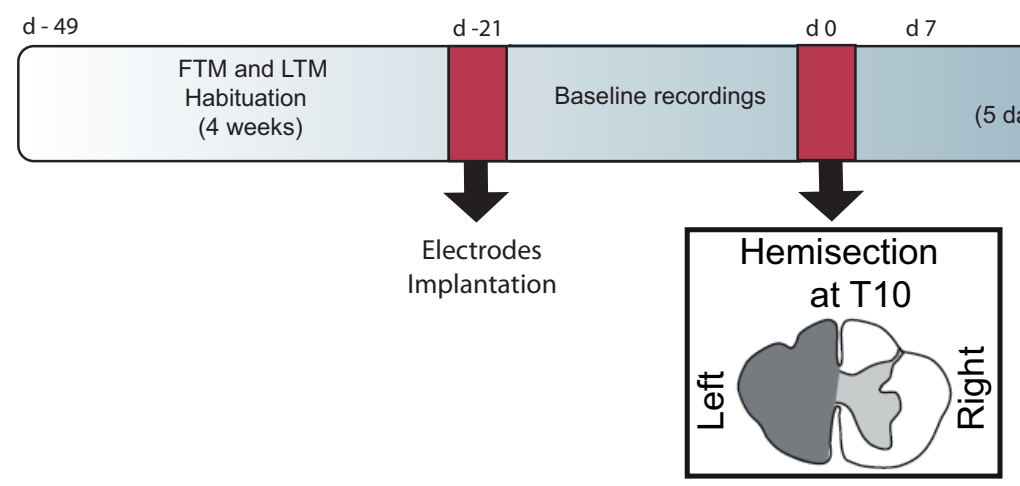

Figure 1. Schematic overview of the LTM setup and experimental protocol. $A$, Left side view of a cat walking on the LTM belt (arrows below the belt indicate the direction of movement) where measurements and spacing between the rungs are shown. Pairs of bipolar EMG wires are implanted into various muscles of the hindlimbs and forelimbs (only one pair is represented here for each limb on the left side) and soldered to a multipin connector cemented to the skull. A digital time code (Society of Motion Picture and Television Engineers) is used to synchronize video and EMG recordings. Reflective markers are placed at the pivotal point of the various joints and the angles measured according to the kinematic model represented. $\boldsymbol{B}$, After a period of habituation of 4 weeks, a left hemisection of the spinal cord was performed at T10. Four days after the spinal lesion, cats were trained during $30 \mathrm{~min}, 5 \mathrm{~d}$ a week to walk on the LTM or on the FTM, and were recorded once every week for $42 \mathrm{~d}$. At the end of the experimental series, cats were prepared to record fictive locomotion, but the results are not discussed here.

Step length. Step length was calculated using the foot contact, which was tagged manually on the first frame when the foot contacted the treadmill surface or the rungs. The distance traveled by the toe between two successive paw contacts of the same limb was defined as the step length.

Angular excursions. For each session, joints angles of the hip, knee, ankle, and MTP joints (see Fig. 1A) and foot lifts/contacts were reconstructed from video images. Then, kinematics was reconstructed from the $x$ - $y$ coordinates of each marker using custom-made software allowing the calculation of the joint angular displacement and the display of the joint excursions. The kinematic model used (direction of flexion/extension for each joint) is detailed in Figure $1 A$. Examples are illustrated in Figure $2 A-C$ showing joint flexion represented by a downward deflection of the traces for each joint. Figure $2 D$, on the other hand, represents the frame-by-frame reconstruction of the videos allowing to visualize the overall movement of the limbs in a stick figure format achieved by connecting each joint sequentially.

Paw trajectory. To assess more precisely the trajectory of the toe in the sagittal plane throughout the swing phase, the $x-y$ coordinates of the toe reflective marker were extracted from the video data to obtain a normalized (in relation to the $x$-axis and with respect to the hip point) average trajectory of the toes in a $2 \mathrm{D}$ plane. This normalization thus allowed to directly compare trajectories when walking on the FTM or LTM before and after spinal hemisection.
Coordination between forelimbs and hindlimbs. The coordination between forelimbs and hindlimbs (i.e., homolateral coordination) and between hindlimbs or both forelimbs (i.e., homologous coordination) was evaluated in the following manner: the period of stance was normalized to 1 and measured for individual limbs; 0 indicates the LH foot contact. The beginning and end of the stance period were displayed as colored rectangles (each limb with a specific color) (see, e.g., Fig. 4). A coupling value of 0.5 meant that the paw contact of a limb occurs at $50 \%$ of the step cycle of the reference LH.

"Walking gaits." "Walking gaits" were identified by the periods of the duty cycles, averaged over 15-20 steps, where 2 or more paws were in contact on the belt or on the rungs. The bipod gait (defined as a gait with 2 paws in contact with the ground simultaneously) was divided according to the relationship between paws, as in Hildebrand (1976). A "homolateral" couplet was defined as the period where both forepaw and hindpaw on the same side of the body made contact on the treadmill at the same time, whereas a "diagonal" couplet was one in which the paws contacted the treadmill on opposite sides of the body. Tripod gait (periods where 3 paws were in contact with the treadmill or rungs) were divided into the following: tripod anterior (both forepaws and one hindpaw) and tripod posterior (both hindpaws and one forepaw). The two combinations were amalgamated in tripod gaits regardless of the side of the paws. 


\section{A}

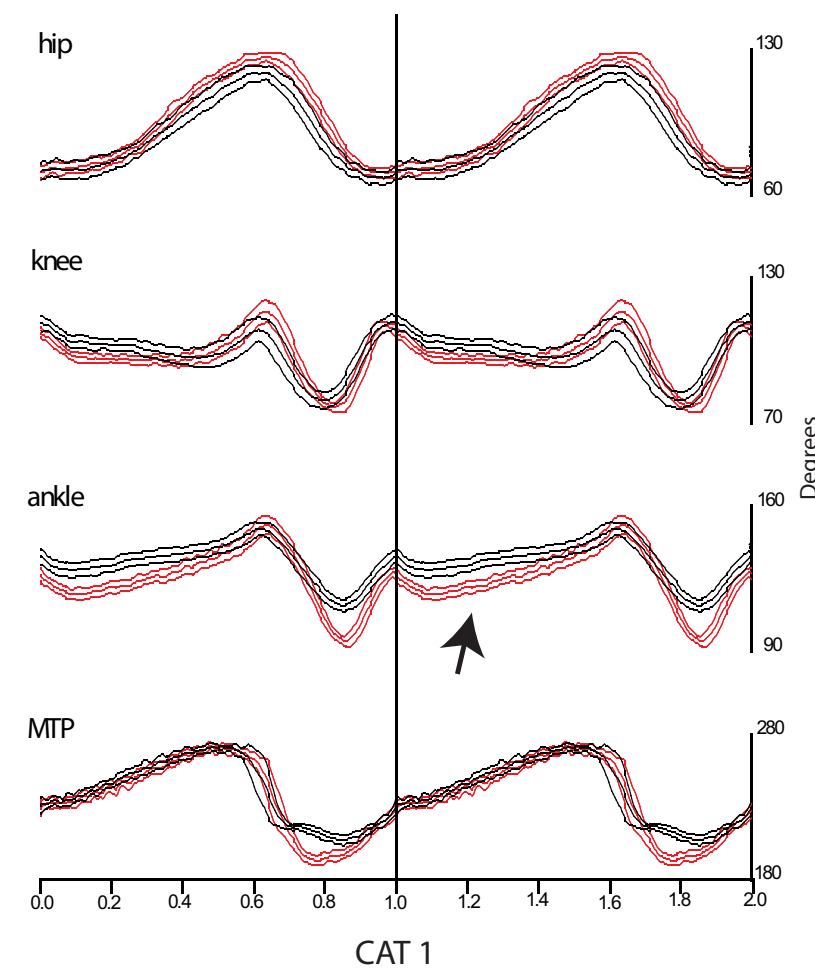

C

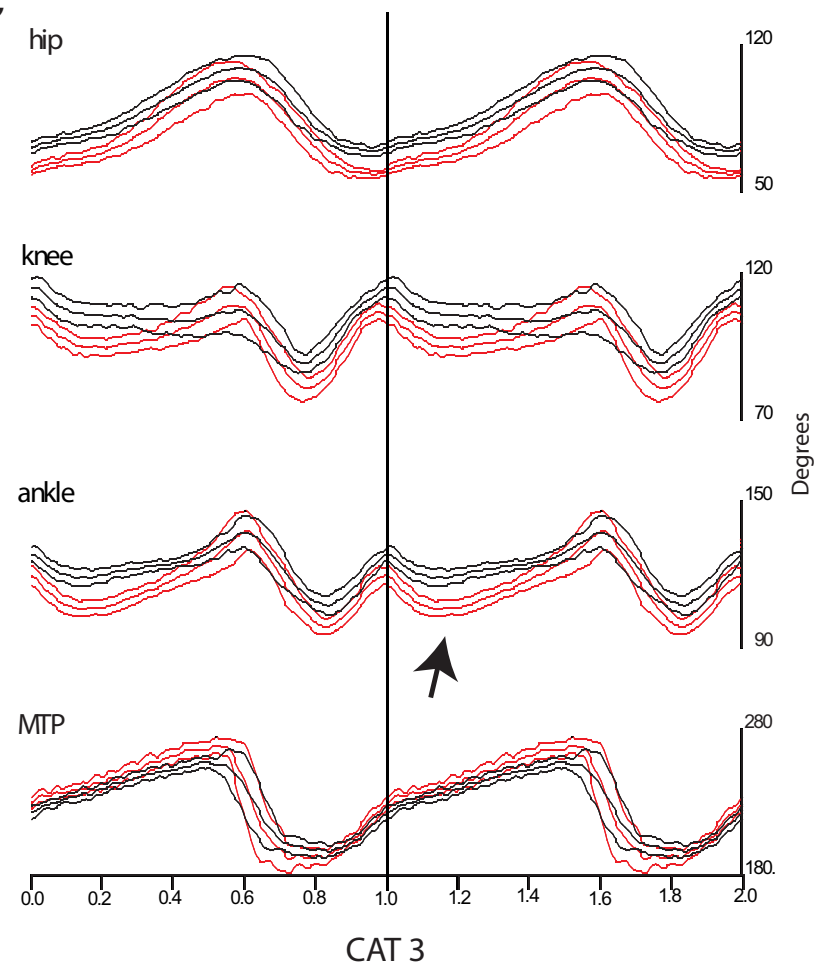

B

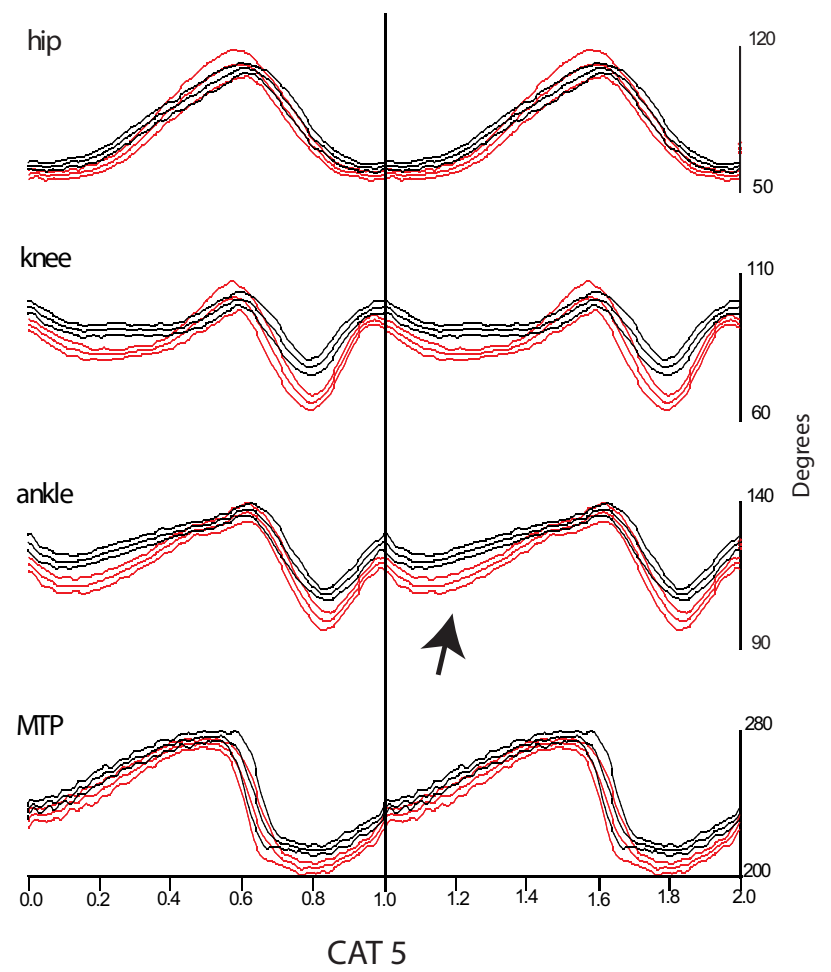

D
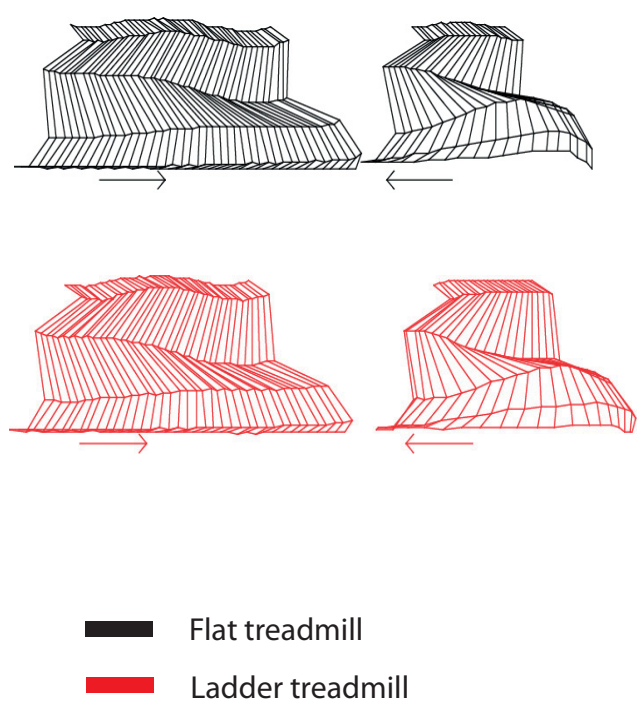

Figure 2. $\quad A-C$, Averaged angular excursion of the hip, knee, ankle, and MTP joints of the LH synchronized on the left paw contact for 3 cats on the FTM (black lines) and on the LTM (red lines). In each group of black and red curves, middle curve indicates the average; curves above and below indicate 1 SD above and 1 SD below the average, respectively. $D$, Stick figures of swing and stance phase from one step cycle of the LH of Cat 1 at $0.5 \mathrm{~m} / \mathrm{s}$ on the FTM and on the LTM.

Foot drag. Foot drag was quantified as the period of time where the dorsal part of the distal phalanx of a given hindpaw dragged over the treadmill belt during the early swing phase. To compare both training methods, cats were regrouped by the similarity of their spinal lesions as evaluated by histological analyses.
EMG burst duration. EMG burst duration was calculated as the time between onset and offset of a single muscle activity. The EMG burst onset and offset were determined using custom-made software that detects the level of EMG signals with precision and average values over several (1520) cycles and were visually verified. Some muscles, such as semitendi- 
nosus or EDB, often have two distinct bursts per cycle, and each could be detected and analyzed separately. The EMG signals were also rectified and integrated allowing measurements of the area under the curve and quantify the amplitude of the activity.

Histology. Six weeks after the hemisection, cats were given a lethal dose of intravenous pentobarbital sodium solution. A piece of spinal cord segment between T8 and L1 (to include the hemisection and nonlesioned tissue) was carefully dissected out and fixed in 10\% PFA for several weeks. The blocks were cryoprotected by successive transfers into increasing concentrations $(10 \%, 20 \%$, and $30 \%)$ of sucrose solution in $0.1 \mathrm{M}$ phosphate buffer for $72 \mathrm{~h}$ at $4^{\circ} \mathrm{C}$. For histological examination, the spinal cord was frozen and $40-\mu \mathrm{m}$-thick coronal sections of a spinal cord segment of $4 \mathrm{~mm}$ centered on the lesion were taken using a cryostat. Every section was mounted on a slide and stained with cresyl violet. Using microscopy, the areas of damage delineated from all the coronal sections in which the lesion was visible (see Fig. 7).

Statistics. Individual kinematic data (step cycle characteristics, angular excursions, foot trajectories, walking gaits) from each animal were averaged from a minimum of 14 consecutive locomotor cycles. Statistical analyses of kinematics were performed using SigmaPlot software (Systat Software). A Student's $t$ test was first used within each animal to determine whether behavior of analyzed kinematic variables comparing FTM and LTM was consistent among cats. For step length and cycle duration, all cats were pooled to obtain mean values. After spinal lesion, values for cycle duration were presented as a percentage of the FTM values obtained in the same cats before spinal lesion. For EMG duration and amplitude, mean values for each cat were normalized according to the FTM values for the same cat. LTM values are presented as a percentage of the FTM values (which represents a fixed value of $100 \%$ ) to reduce bias in voltage values on EMG discharges due to differences in electrode positions or intrinsic muscle differences between cats. For the foot drag, a Student's $t$ test was used to determine whether foot drag was significantly different with FTM or LTM. When data showed non-normal distribution, the nonparametric $U$ test of Mann-Whitney was used, as equivalent of the parametric $t$ test. Two-way ANOVA tests were used to compare groups and for different delays after spinal hemisections. In all figures, statistical significance between conditions is indicated by an asterisk. To compare the performances on the FTM and LTM stepping, a $p$ value of $<0.05$ was considered statistically significant. Results are presented as mean $\pm \mathrm{SE}$.

\section{Results}

Results are presented in three sections. The first describes and compares the kinematics and EMG characteristics of cats with an intact spinal cord walking on the FTM and on the LTM. The second section details how cats with a unilateral spinal hemisection walk on the FTM and LTM. The third section evaluates the effects of locomotor training on FTM and LTM over a period of $42 \mathrm{~d}$ after the hemilesion on the locomotor pattern of the FTM. Figure $1 B$ illustrates graphically the timeline of the complete paradigm. Cats $1-3,5-7$ were trained on the LTM, whereas Cats $8-10$ were trained on the FTM.

\section{Locomotion of cats with an intact spinal cord on an FTM or on an LTM}

Experiments were conducted in 6 adult cats with an intact spinal cord, which could maintain several consecutive and regular steps at $0.5 \mathrm{~m} / \mathrm{s}$. They were habituated daily for 4 weeks to walk alternately on the FTM or the LTM or until they learned both tasks, at which time training sessions were performed only at every 2 or $3 \mathrm{~d}$. The walking patterns of 15-20 consecutive steps on the FTM and LTM were collected (kinematics and EMGs) on the same day. Therefore, the data represent stable behaviors for each cat in both conditions allowing a direct comparison of EMG and kinematics between both tasks.

The task on the LTM clearly required a visual input because cats relied heavily on seeing the coming rungs through the clear Plexiglas at the front of the treadmill enclosure. When simply
Table 1. Total amplitude of angular excursions of the hindlimb joints in degrees ${ }^{a}$

\begin{tabular}{|c|c|c|c|c|c|}
\hline \multicolumn{2}{|c|}{ Cat 1} & \multicolumn{2}{|c|}{ Cat 2} & \multicolumn{2}{|c|}{ Cat 3} \\
\hline FTM & LTM & FTM & LTM & FTM & LTM \\
\hline \multicolumn{6}{|l|}{ Hip joint } \\
\hline $53 \pm 2$ & $59 \pm 2^{* * *}$ & $54 \pm 3$ & $69 \pm 3^{* * *}$ & $43 \pm 3$ & $48 \pm 7^{*}$ \\
\hline \multicolumn{6}{|l|}{ Knee joint } \\
\hline $36 \pm 3$ & $42 \pm 3^{* * *}$ & $31 \pm 5$ & $44 \pm 3^{* * *}$ & $26 \pm 4$ & $33 \pm 5^{* * *}$ \\
\hline \multicolumn{6}{|l|}{ Ankle joint } \\
\hline $40 \pm 3$ & $58 \pm 2^{* * *}$ & $48 \pm 7$ & $66 \pm 4^{* * *}$ & $35 \pm 5$ & $44 \pm 6^{* * *}$ \\
\hline \multicolumn{6}{|l|}{ MTP joint } \\
\hline $68 \pm 2$ & $82 \pm 4^{* * *}$ & $79 \pm 3$ & $87 \pm 5^{* * *}$ & $69 \pm 5$ & $86 \pm 11^{* * *}$ \\
\hline \multicolumn{2}{|c|}{ Cat 5} & \multicolumn{2}{|c|}{ Cat 6} & \multicolumn{2}{|c|}{ Cat 7} \\
\hline FTM & LTM & FTM & LTM & FTM & LTM \\
\hline \multicolumn{6}{|l|}{ Hip joint } \\
\hline $50 \pm 2$ & $47 \pm 2^{* * *}$ & $38 \pm 2$ & $43 \pm 1^{* * *}$ & $47 \pm 2$ & $58 \pm 3^{* * *}$ \\
\hline \multicolumn{6}{|l|}{ Knee joint } \\
\hline $25 \pm 2$ & $37 \pm 3^{* * *}$ & $27 \pm 2$ & $28 \pm 2$ & $31 \pm 1$ & $34 \pm 2^{* * *}$ \\
\hline \multicolumn{6}{|l|}{ Ankle joint } \\
\hline $31 \pm 1$ & $33 \pm 2^{*}$ & $44 \pm 4$ & $44 \pm 3$ & $31 \pm 2$ & $46 \pm 4^{* * *}$ \\
\hline \multicolumn{6}{|l|}{ MTP joint } \\
\hline $68 \pm 4$ & $75 \pm 4^{* * *}$ & $86 \pm 6$ & $87 \pm 5$ & $79 \pm 5$ & $84 \pm 4^{* * *}$ \\
\hline
\end{tabular}

inserting a cardboard in front to block the view, the cat moved back with the treadmill to ensure it could see 3 or 4 rungs ahead.

\section{Step cycle characteristics}

Cats were gently encouraged to walk on the LTM, making sure that they felt comfortable placing consecutively all the feet on the rungs for several successive cycles. It is important at this early stage to avoid adverse events that could compromise the rest of the experiments. The speed of the treadmill was gradually increased and cats could comfortably correct their stepping when one or more feet were placed between rungs. The distance between rungs was fixed for each cat of different sizes to accommodate their most comfortable step length. The averages of step length and step duration while walking on the FTM or the LTM were not statistically different for the 6 cats compared on the same day and at the same walking speed. Even lengths and duration for swing and stance on the left and right sides were not statistically different (paired $t$ test, $p>0.05$ ). This was deemed important to ensure that changes in angular excursion or EMG activity were related to the demands of the walking tasks on the LTM and FTM and not to changes in the basic locomotor characteristics.

\section{Angular excursions and foot trajectory}

Figure 2 shows the angular excursions of the LH (facing the camera) during normal stepping on an LTM (red lines) and on the FTM (blacklines) for 3 cats. Angular excursions were superimposed and synchronized on the contact of the left hindpaw to visually compare the two tasks. Although the overall angular excursions on the LTM and FTM followed a similar shape (which was somewhat unexpected given the different demands of both tasks), there were significant differences in the maximum amplitude for all angles in all cats, except more distal joints in Cat 6 (Table 1). The most important change was an increase in knee and/or ankle peak flexion on the LTM. This occurred in the middle and later part of the swing phase, before contacting the rungs and right at the beginning of the stance phase where a yield at the ankle started and was prolonged for the greatest part of the stance phase (for yield at the ankle for the 3 cats, see Fig. $2 A-C$, black 


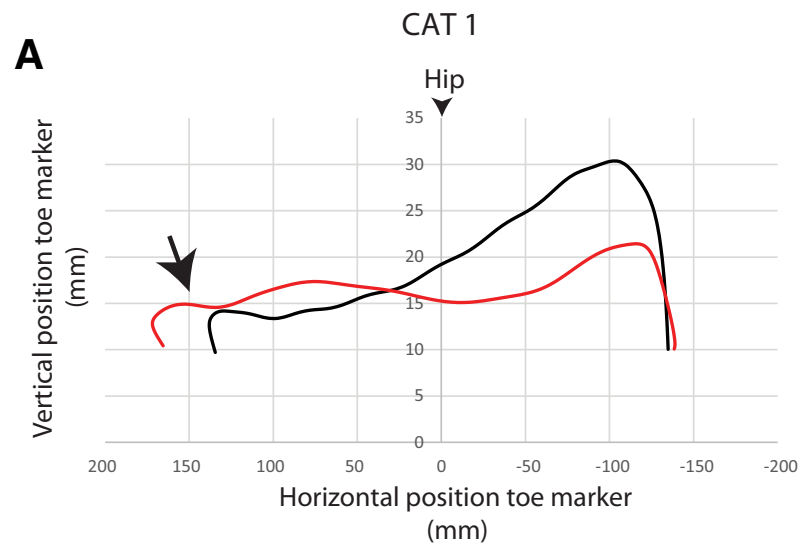

CAT 2

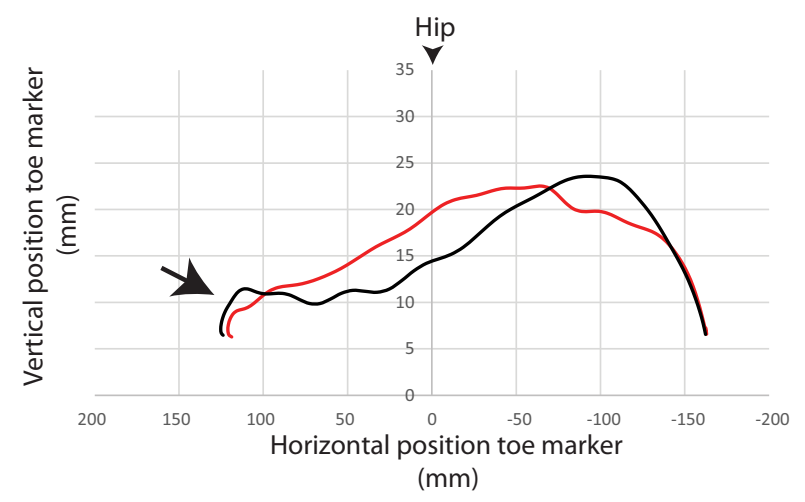

CAT 5

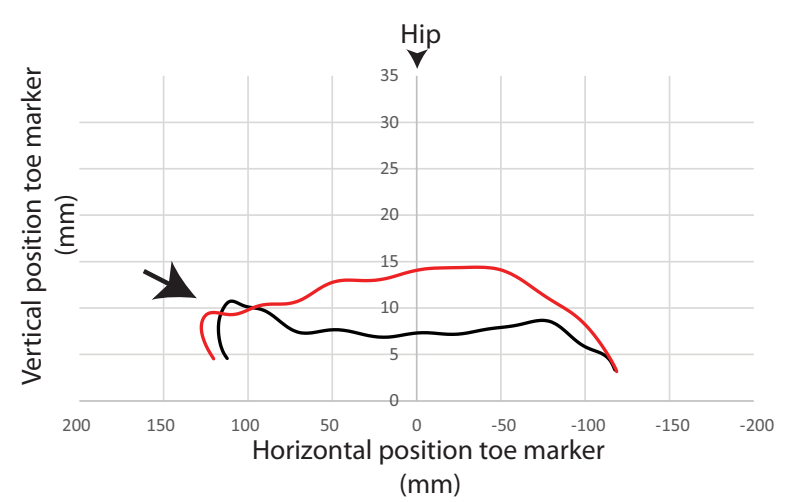

B

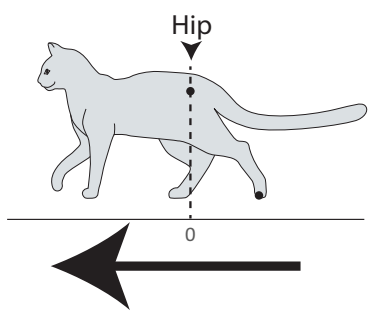

Flat treadmill

Ladder treadmill

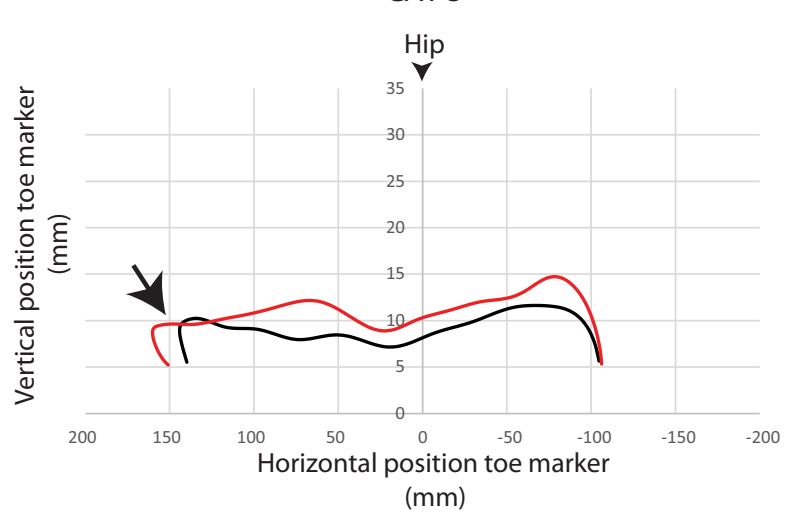

CAT 6

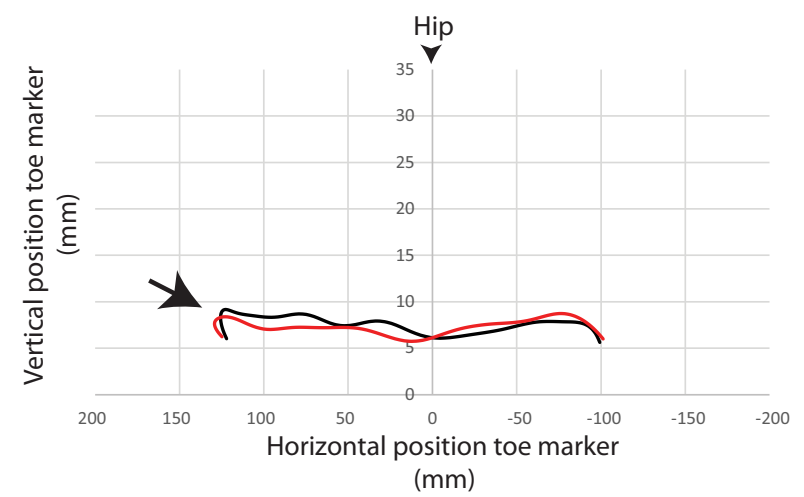

Figure 3. A, Graphical 2D representations of left toe trajectories during FTM (black lines) and LTM (red lines) stepping (17-20 step cycles) in Cats 1, 2, 3, 5, and 6. A "hook" shape (black arrow) was seen before the foot contact in both FTM and LTM tasks. $\boldsymbol{B}$, Representation of the walking direction and localization of the reflective marker on the left toe used as reference to calculate its trajectory. Point 0 indicates the vertical projection of the hip joint marker with the ground.

arrows). Stick figures derived from sequences on FTM and LTM were quite similar (Fig. 2D, Cat 1).

Sometimes, combined small changes in amplitude in the excursion of proximal joints can lead to significant distal changes, such as in paw trajectories. Fig. 3 shows that the overall horizontal distance of the toe marker, from foot lift to foot contact, measured with respect to the hip position set at zero was remarkably similar in both walking tasks. However, in 3 of 5 cats, the vertical displacement of the toes on the LTM (red lines), especially at around the middle of the swing ( $0 \mathrm{~mm}$ on abscissa), was increased. For example, in Cat 5, the combination of small angular changes in the knee and ankle (Fig. $2 B$ ) resulted in a higher ver- tical displacement of the toe during swing of $\sim 7 \mathrm{~mm}$ when walking on LTM compared with FTM.

Figure 3 also shows that, before foot contact, the marker at the tip of the toes was displaced slightly upward before being brought downward and slightly backward just before contact with either the surface of the FTM or LTM rungs. This gave rise to a characteristic "hook" shape terminal trajectory, suggesting a precise control of the toes just before contact. This was seen in 5 of 6 cats (Fig. 3, arrows).

\section{Interlimb coupling and walking gaits}

Figure 4 illustrates the interlimb coupling in both walking conditions using gait diagrams as well as footfall patterns (top mid- 
A Gait diagram

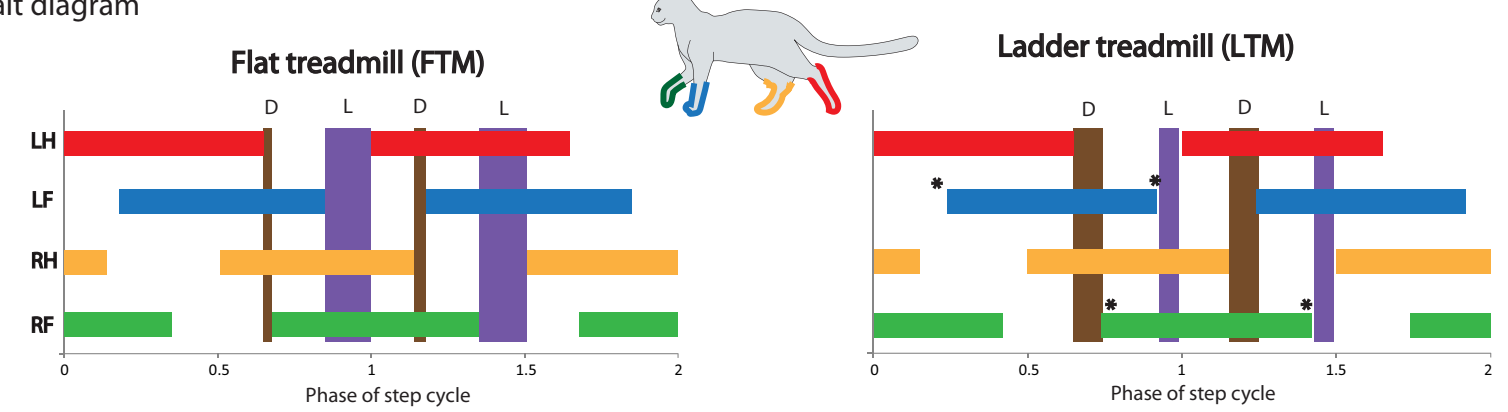

B Footfalls patterns

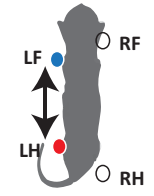

Diagonal/lateral couplets FTM

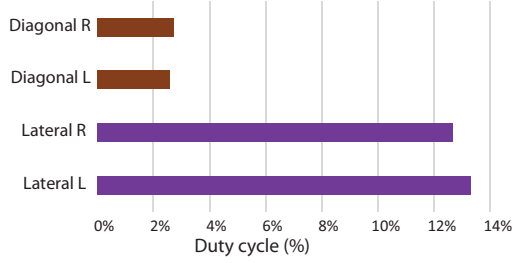

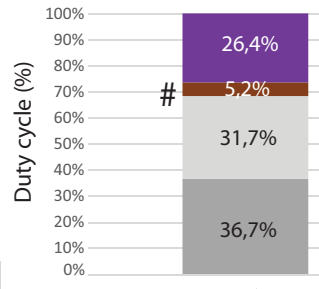

FTM

anterior posterior
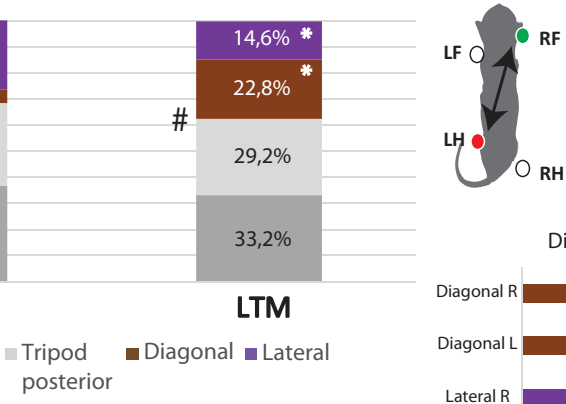

Diagonal/lateral couplets LTM

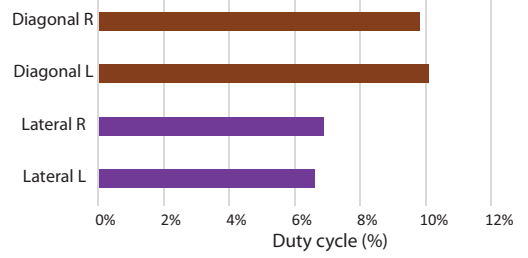

Figure 4. Interlimb coordination during FTM and LTM locomotion. $A$, Gait diagrams showing the stance phases of the LH (red), LF (blue), RH (yellow), and RF (green). Intervals between the stance phases represent the swing phase. The color code follows that of the cat figurine. Vertical rectangles represent periods of diagonal ( $D$, brown) or lateral ( $L$, violet) coupling between the hindlimbs and forelimbs. The cycle starts with the LH contact (time 0 ), and the cycle is repeated from time 1 to time 2 to ensure a proper representation of transitions at around foot contact. ${ }^{*} p<0.05$, FTM versus LTM. $\boldsymbol{B}$, Footfall patterns illustrate the percentage of the duty cycle with different feet on the ground during FTM and LTM walking. Gray rectangles represent periods where three feet are on the ground: either 2 hindlimbs and 1 forelimb (tripod posterior) or 2 forelimbs and 1 hindlimb (tripod anterior). Violet rectangles represent periods in violet in $\boldsymbol{A}$ where the two limbs on one side are coupled (lateral coupling). Brown rectangles represent diagonal coupling. Hashtags in the FTM column indicate a statistical difference between the diagonal and lateral coupling. Hashtag in the LTM column indicates a statistical difference between diagonal couplings in both conditions. The figurines adjacent to the distribution of interlimb coupling modes illustrate the predominant forelimbhindlimb coupling in both tasks. The distribution of interlimb coupling is pooled for left and right coupling. Bottom for both FTM and LTM, details of the type of coupling: diagonal right or left and lateral right or left.

dle, inset, limbs color-coded); the sequence of foot contacts is $\mathrm{LH}$ (red), left forelimb (LF, blue), right hindlimb ( $\mathrm{RH}$, yellow), and right forelimb (RF, green), which is the usual pattern of cat locomotion. This sequence was maintained for both FTM and LTM. There was a strict alternation ( 0.5 phase) between homologous forelimbs and hindlimbs. However, the hindlimb-forelimb couplings were changed on LTM so that the forelimbs contacted the rungs later than they do on the FTM. This change in homolateral coupling resulted in a difference in the number of paws simultaneously in contact with the rungs on the LTM or the FTM. For example, the time interval between the lift of LF (blue) and LH contact (red) is on average larger on the FTM than on the LTM, as will be detailed below. This corresponds to periods where only the RF and RH (homologous limbs) are in contact with the treadmill.

In Figure $4 A, B$, vertical bars (violet and brown) represent periods (expressed as percentage of the step cycles) where two feet are in contact with the belt (brown for diagonal gait, violet for lateral gait). The footfall patterns (Fig. 4B) show that bipod couplings were quantitatively different in FTM and LTM: on the FTM, the percentage of the duty cycle devoted to lateral couplets (violet bars) is $26.4 \%$, whereas, on the LTM, diagonal couplets (thick brown bars) predominate $(22.8 \%)$ (Student's $t$ test, $p<$ 0.05 ). Therefore, during LTM, more time was spent with three feet in contact with ground than during FTM walking, probably to increase stability in the more challenging walking task.
The blocks of horizontal bars below the footfall patterns for FTM (on the left) and LTM (on the right) illustrate that diagonal and lateral couplets are symmetrical on both sides despite the fact that diagonal couplings are more frequent in LTM conditions.

\section{EMG activity}

There were some consistent changes (in amplitude and/or time profile) in the EMG activity of specific muscles. Figure 5 illustrates some of these changes for the $\mathrm{LH}$ and forelimb. EMGs are rectified, superimposed, and synchronized to the contact of the respective limb (black lines indicate FTM; red lines indicate LTM). Mean amplitude and mean duration of EMG bursts for each implanted muscle in all cats, expressed as a percentage of respective baseline values, are shown in Figure 6.

\section{Hindlimb flexor muscles}

The semitendinosus, a bifunctional muscle acting as a knee flexor and a hip extensor, has a particular activity pattern consisting of two bursts during the swing phase. The first and largest burst has a sudden onset occurring around paw lift; and a second shorter burst, which is not always present at very low speeds, precedes paw contact (J. L. Smith et al., 1993).

Although the duration of the first semitendinosus burst (Fig. 6C) showed no significant difference between the two tasks, the overall amplitude of the "integrated" first burst was statistically larger (Fig. $6 A$ ) on the LTM (paired $t$ test; $p<0.05$ ). More re- 
A

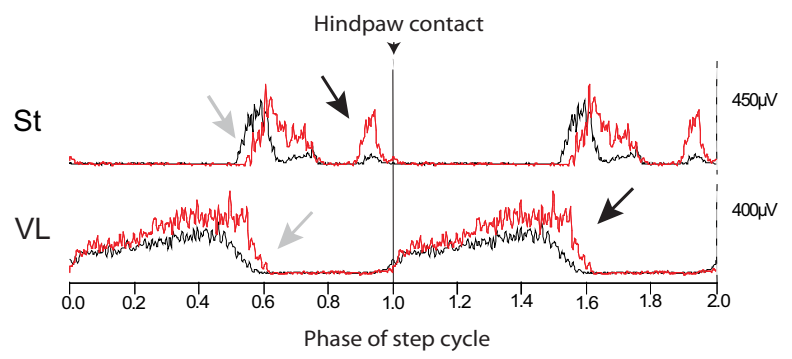

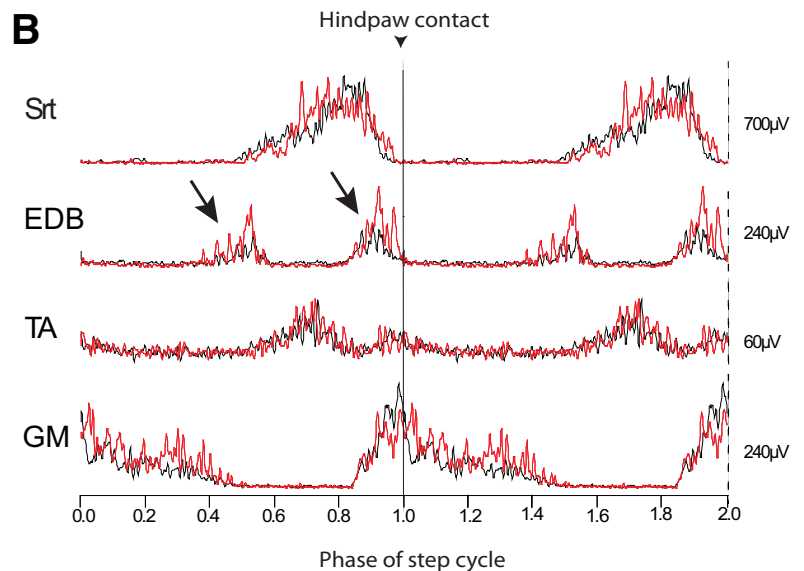

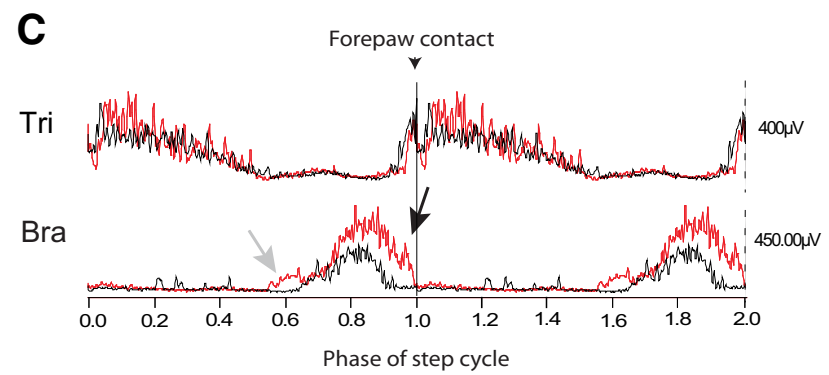

- Flat treadmill

Ladder treadmil

Figure 5. Examples of rectified and averaged activity in implanted muscles of 15 steps on the FTM (black lines) superimposed on 20 steps on the LTM (red lines) from Cat 1 ( $\boldsymbol{A}, \boldsymbol{C}$ ) and Cat 3 ( $\boldsymbol{B}$ ). Averages were synchronized on the contact of the left forepaw or the left hindpaw facing the recording camera that corresponds to the muscles of the same limb, as indicated by the continuous vertical line at Phase 1. Black arrows indicate the main differences in the EMG profiles between FTM and LTM. Gray arrows indicate phase shifts in the LTM task compared with the FTM. St, Semitendinosus; Srt, sartorius; TA, tibialis anterior; Tri, triceps brachii; Bra, biceps brachii.

A
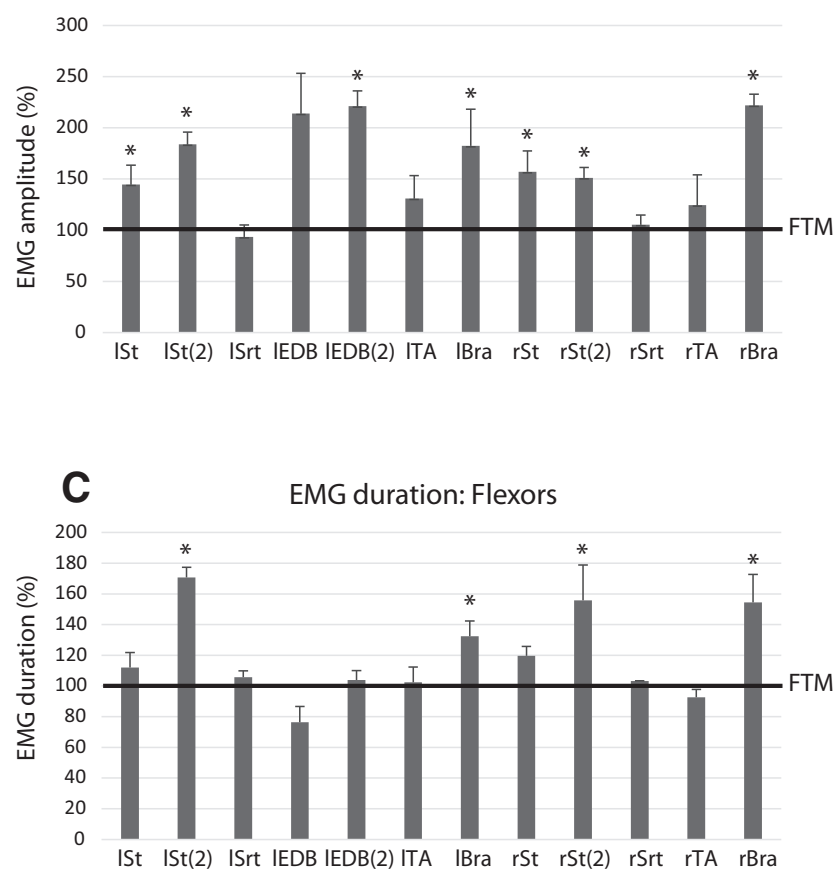

B
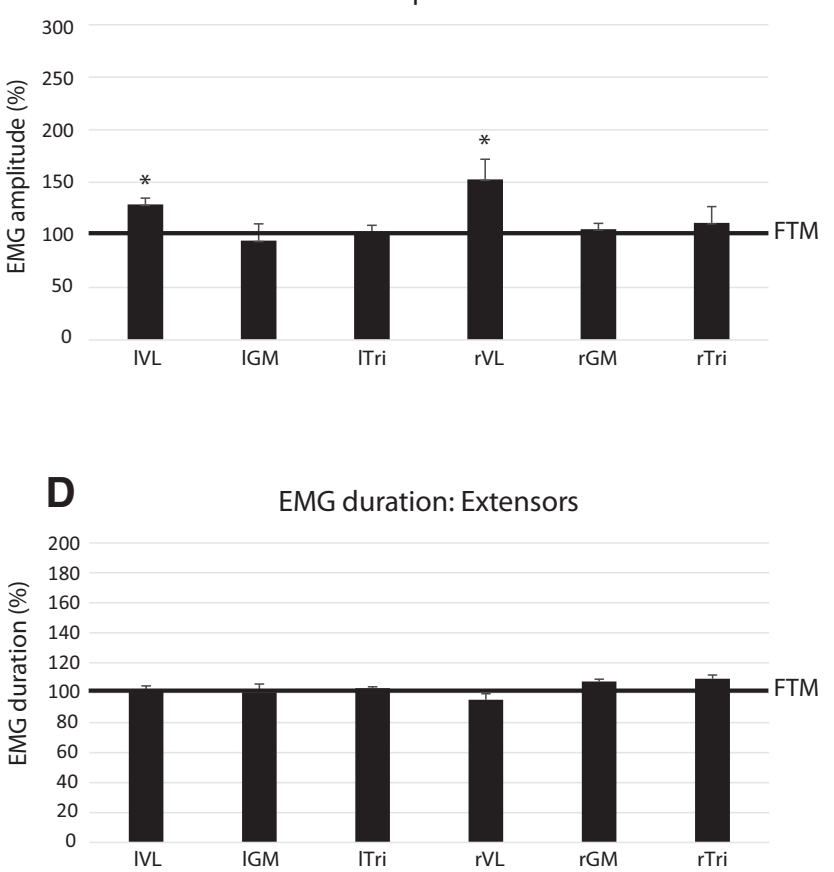

Figure 6. EMG amplitude and duration on the LTM task for implanted flexor $(\boldsymbol{A}, \boldsymbol{C})$ and extensor $(\boldsymbol{B}, \boldsymbol{D})$ muscles for all cats with an intact spinal cord. LTM values are presented as a percentage of the FTM values (which represents a fixed value of 100\%, thick black line). I, Left; r, right; St, semitendinosus; St(2), semitendinosus second burst; Srt, Sartorius; EDB(2), EDB second burst; TA, tibialis anterior; Tri, triceps brachii; Bra, biceps brachii. *Statistical differences between FTM and LTM condition for each muscle. 
Ladder treadmill trained cats

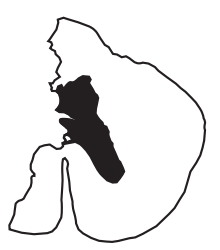

Cat 1

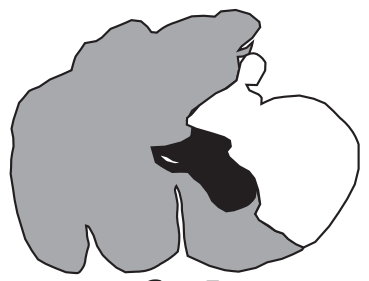

Cat 5

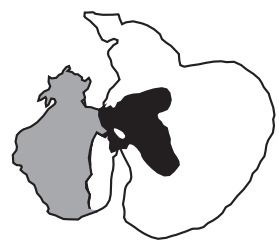

Cat 8

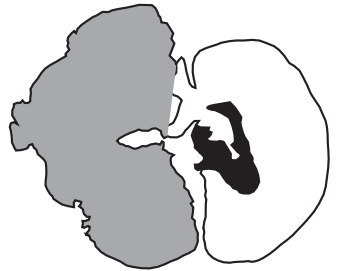

Cat 2

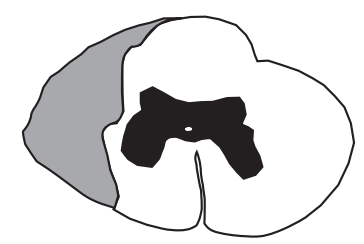

Cat 6

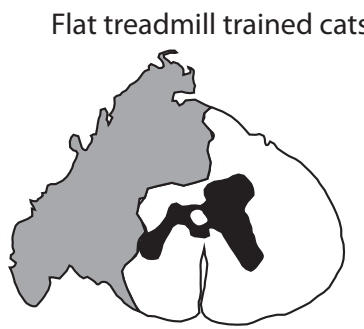

Cat 9

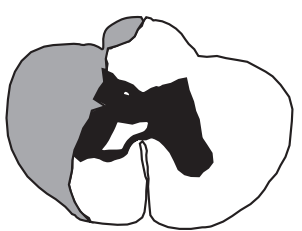

Cat 3

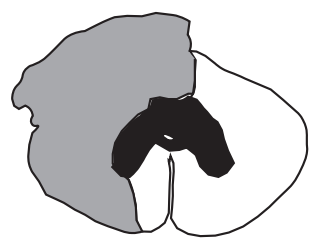

Cat 7

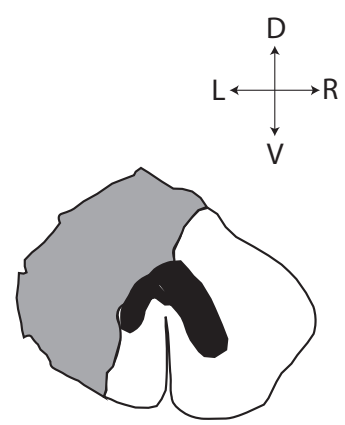

Cat 10

Figure 7. Schematic drawings of the hemisections at T10 of individual cats taken from Nissl slices. Gray represents damaged tissue. Black represents gray matter. D, Dorsal; $V$, ventral; $L$, left; $R$, right.

markably, the amplitude and duration of the second semitendinosus burst just before foot contact (Figs. $5 \mathrm{~A}$, black arrow) were almost doubled on the LTM compared with the FTM (paired $t$ test; $p<0.05$ ). Tibialis anterior and sartorius muscles showed no changes in shape, duration, and amplitude on the LTM in 3 of the cats in which the recordings were very clear.

EDB muscle has a special activity in locomotion. It is activated during the first extension phase, but it does not relax immediately after the foot contact as the rest of the flexors. For simplicity, we refer to it in this manuscript as a "flexor." Actually, EDB presents 2 bursts of activity (Fig. 5B, black arrows) synchronized in time with those of the semitendinosus (Engberg and Lundberg, 1969). We found that EDB's first burst showed a more variable discharge pattern during FTM locomotion (i.e., there were cycles where this first burst was absent). Therefore, the average shown in Figure $6 \mathrm{~A}$ might be misleading because it includes cycles where EDB was present, very small, or absent. By contrast, on the LTM, EDB activity was always present and showed the characteristic double burst. The second EDB burst, which was always present in both conditions, showed a significant increase in amplitude on the LTM (paired $t$ test, $p<0.05$ ) but showed no change in duration.

\section{Hindlimb extensor muscles}

The activity of the knee extensor muscle VL showed a significant increase in amplitude (Fig. 5, VL traces, black arrow) on the LTM (paired $t$ test, $p<0.05$ ) presumably to increase support during the stance phase while the fellow hindlimb swings forward. There were no significant changes in duration despite a somewhat later offset of the burst in this example. The increase in VL activity probably helps to offset the clear yield seen at the ankle (Fig. $2 A, 3$ arrows). The ankle extensor GM muscle showed no significant change of amplitude or duration (Fig. $6 B, D$ ). In agreement with previous experiments (Engberg and Lundberg, 1969; Gorassini et al., 1994) illustrating that EMG activity before foot contact is centrally preprogrammed, the precontact activity of GM was not apparently changed in the LTM condition.

EMG activities in many muscles also showed some time shifting (i.e., changes in the onsets and offsets of bursts, Fig. $5 A$ ). For instance, the onset of the first burst in semitendinosus occurred earlier (backward shift) during LTM (Fig. 5A, gray arrow) but ended at the same time as on the FTM. The VL bursts started at the same time but ended somewhat later.

\section{Forelimb flexor and extensor muscles}

The amplitude and duration of biceps brachii muscle activity were both significantly increased in LTM stepping (Figs. $5 C, 6 A, C$, black arrows) compared with FTM (paired $t$ test; $p<0.05$ ). Biceps brachii muscle also showed an earlier discharge on the LTM (Fig. 5C, gray arrow). The larger and somewhat delayed burst may correspond to the later contact of the forelimb during LTM walking (see Interlimb coupling and walking gaits). Triceps brachii muscle's burst amplitude and duration showed no significant changes on the LTM (Figs. 5C, 6B,D).

\section{Locomotion on the FTM of cats with unilateral hemisection}

The second aim of the project was to investigate the capacity of cats with a spinal hemisection to walk LTM using their remnant spinal pathways.

Hemisected cats were divided into two groups. For 6 weeks, 6 cats (Cats 1-3, 5-7) were submitted to training exclusively on the LTM, whereas 3 other cats (Cats $8-10$ ) were trained exclusively on the FTM. Once a week after the hemisection, the stepping performance of all cats was recorded on the FTM (the "outcome"). This allowed a direct comparison of kinematics and EMG activities for both groups submitted to different training regimens.

\section{Histological assessment}

Figure 7 shows outlines of coronal spinal cord sections from histological microscopic observations. White areas represent the spared nondamaged white matter, whereas black represents the gray matter. Gray represents lesioned matter, whereas void areas represent tissue lost in histological processing. In all cats, important damage of the left side, sparing only a small portion of the ventral quadrant except for Cats 2 and 5, was observed. Cat 6 showed the smallest lesion with all gray matter spared bilaterally, whereas Cat 5 showed the largest lesion encroaching on the right side. Also, Cats 1 and 8 presented quite similar lesions and will be used for some comparisons. 
A CAT 3 HEMI14 LTM walking

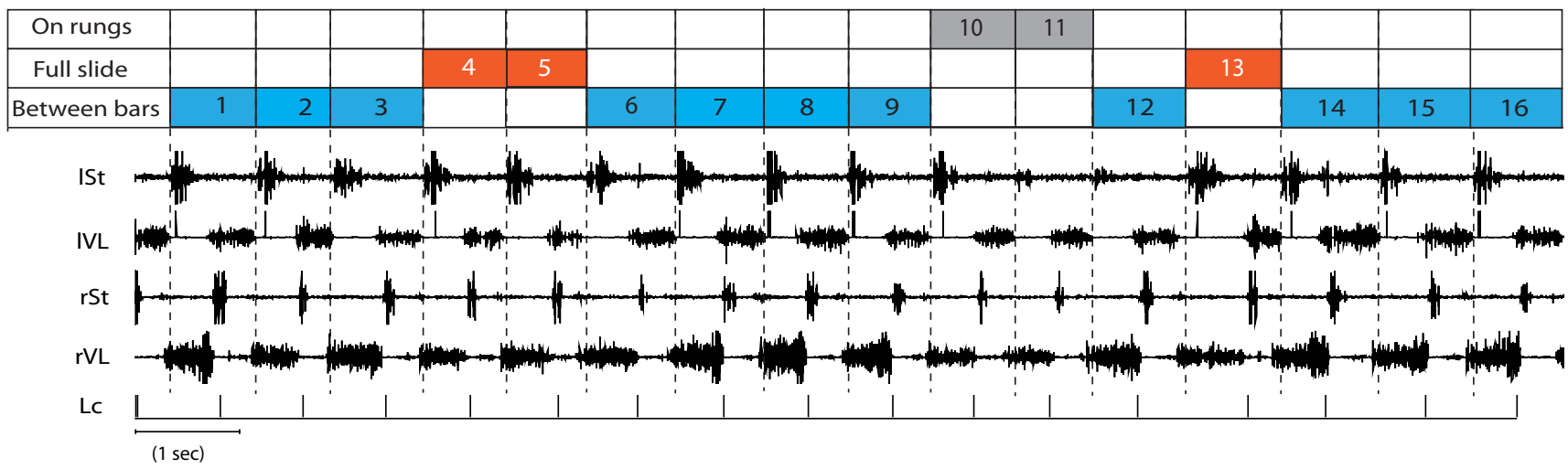

B

CAT 7 HEMI14 LTM walking

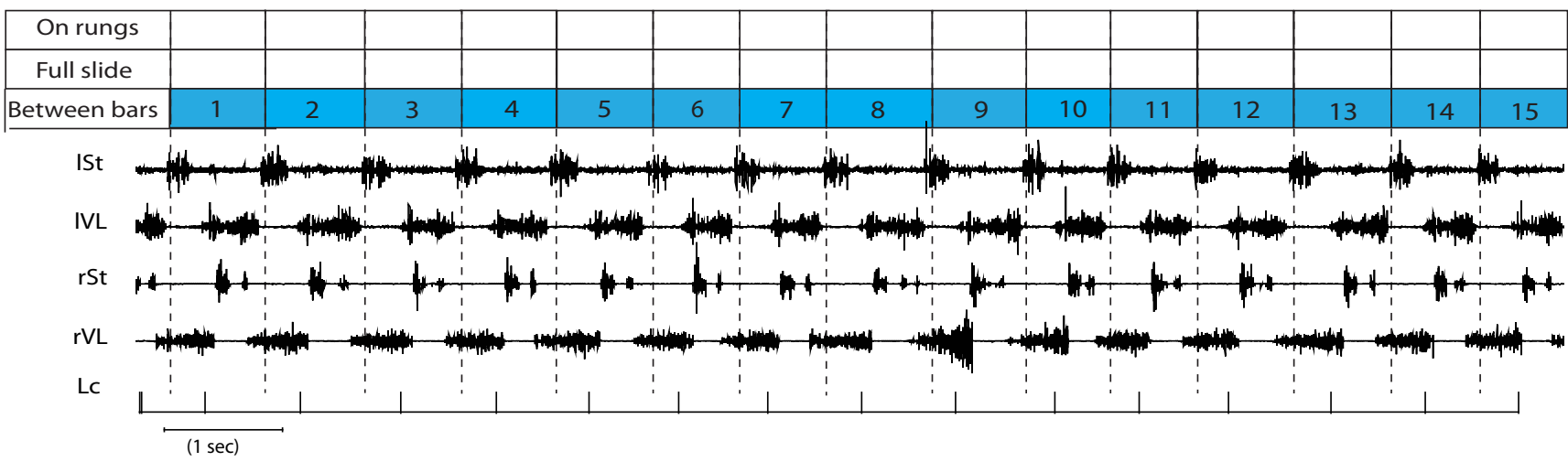

C

CAT 7 HEMI42 LTM walking

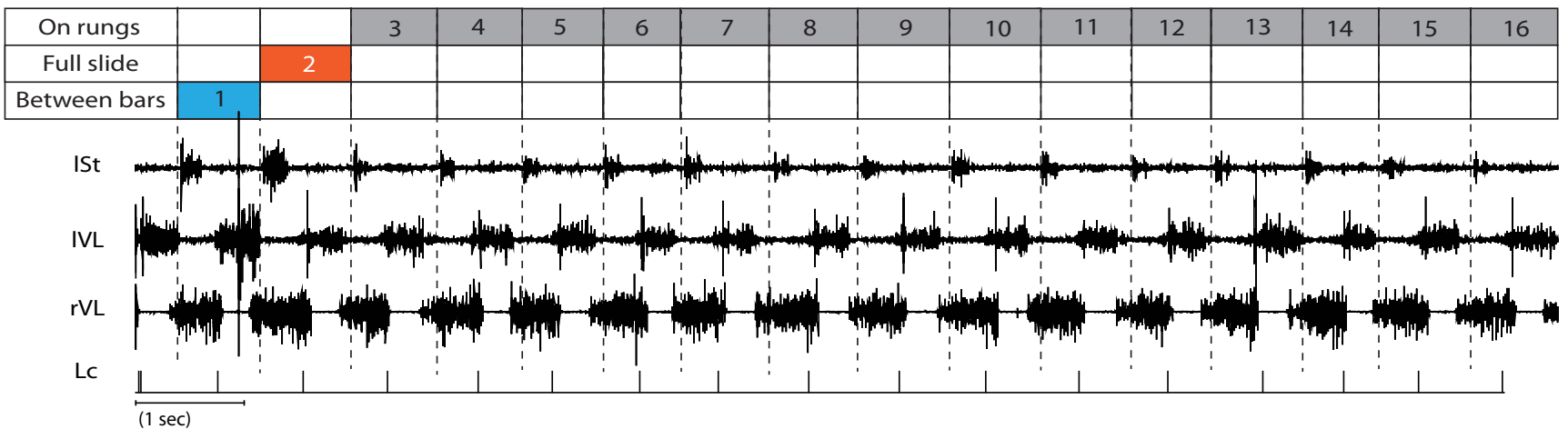

Figure 8. Examples of raw EMG activity in Cats 3 and 7 during LTM stepping 14 or $42 \mathrm{~d}$ after spinal hemisection. Numbers inside boxes on the top of the EMGs indicate each step associated with each form of left hindpaw placement observed during LTM walking sessions: on the rungs (gray boxes); full slide (orange boxes), the left paw contacted the rung, but it glided to the bottom of the space between rungs; and between bars (blue boxes). Each step cycle (numbers inside boxes) was synchronized with the beginning of a semitendinosus burst of the selected sequence and ended on the beginning of the next semitendinosus burst. Colored markers inside the EMG boxes indicate each step cycle. $\boldsymbol{A}$, Cat walks between the rungs, on the rungs, or with a full slide. $\boldsymbol{B}$, Cat 7 could walk stably between the bars or, as in C, between the rungs. St, Semitendinosus; LC, left contact.

Stepping and recovery of paw placement on rungs during LTM training

Locomotion on the LTM varied among cats and, with time after the hemisection on the left side, on the amount of locomotor training. The description will focus mainly on the LH innervated by spinal segments below the lesion. Figure 8 illustrates three examples of locomotor sequences on the LTM. Figure $8 \mathrm{~A}$ represents Cat 3 ( $14 \mathrm{~d}$ after lesion, walking irregularly with the $\mathrm{LH}$ on the rungs, between the rungs, or sliding on one rung to contact the treadmill between two rungs). Fig. 8 shows Cat 7, $14 \mathrm{~d}$ after hemisection, with its LH walking comfortably and regularly be- tween rungs. The regularity of the stepping indicates that this was a precise voluntary modification even though the foot contact was not on the rungs themselves (i.e., this pattern was not random). Figure $8 C$ shows the same cat, $42 \mathrm{~d}$ after hemisection, walking mainly on the rungs. All the cats, at all times, place all 3 other feet on the rungs so that deficits were only seen in the hindlimb on the side of the hemisection. As seen in Fig. 8 (top), cats could adopt such a limping walking pattern (left hindfoot between rungs and all other feet on the rungs) without apparent discomfort, although, by periods, cats could alternate between walking on the rungs or between the rungs. 
A CAT 614 days after spinal lesion
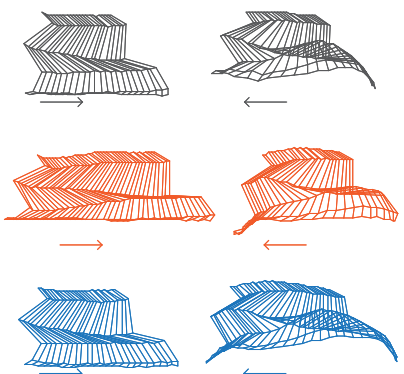

On rungs (4 steps)
Full slide (5 steps)

Between rungs (10 steps)
CAT 314 days after spinal lesion
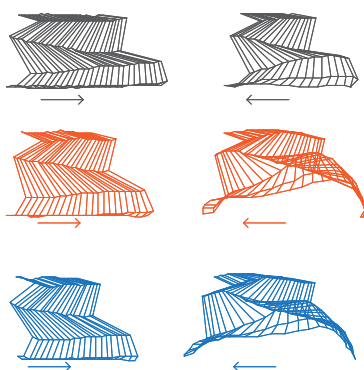

On rungs (2 steps)

- Full slide (3 steps)

Between rungs (11 steps)
CAT 721 days after spinal lesion

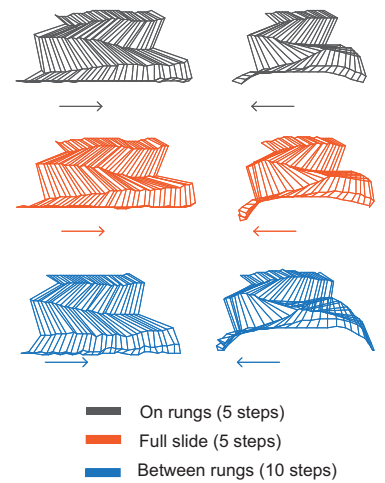

Recording

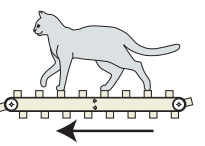

B

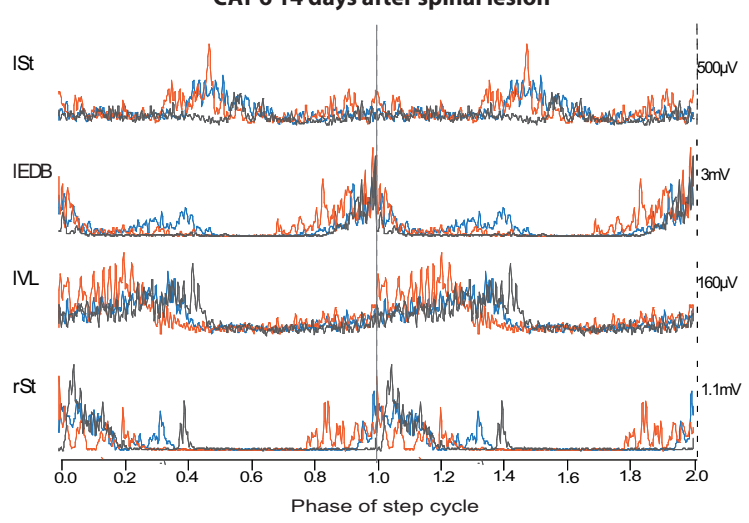

CAT 314 days after spinal lesion

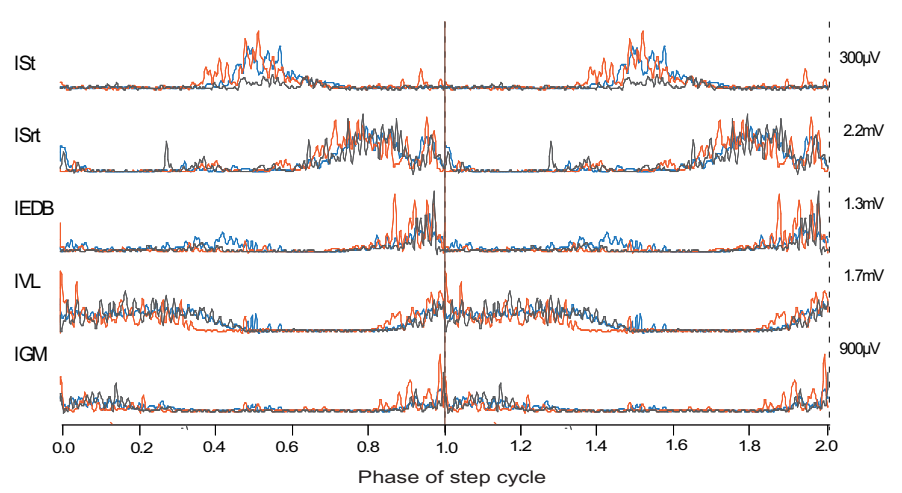

Figure 9. A, Stick figures of swing and stance phase from one representative step cycle of the LH of Cats 6, 3, and 7 at 14 and $21 \mathrm{~d}$ after spinal hemisection walking on the LTM. Paw placement patterns: on the rungs (gray bars), full slide (orange bars), and between bars (blue bars) as well as the number of steps given for each paw placement pattern during the same walking sequence. $B$, Averaged rectified EMG locomotor bursts synchronized on the left paw contact in Cats 6 and 3 at $14 \mathrm{~d}$ after spinal hemisection comparing EMG activity for the selected muscles during the different paw placement patterns observed during LTM walking. Note the increase in semitendinosus while walking below the rungs or with a full slide. I, Left; r, right; St, semitendinosus; Srt, sartorius.

Figure $9 A$ shows kinematic figurines of 3 cats walking on the rungs, sliding on the rungs, or walking between the rungs. Although walking on the rungs requires some alterations of the swing phase (lift of the foot during swing is larger than control), the most dramatic modifications or adjustments are seen when walking between rungs. The toe trajectories illustrate well how the foot makes a large swing to overcome the rung and reach the next inter-rung space. Although not measured directly (because video is taken from the left side), the visual inspection of right limb does not reveal any particular adjustment as it walks regularly on the rungs even though the LH walks between rungs. Figure $9 B$ illustrates the changes in some of the EMGs corresponding to the different gaits. Clearly, the knee flexor semitendinosus increased dramatically when walking between rungs or sliding on the rungs. The knee extensor VL was shorter as the foot slipped on the rung, but it was normal when walking on the rungs or between the rungs.

Fig. 10 compares EMG activity of some flexor and extensor muscles on the LTM before and after hemisection and training. First, the second burst in semitendinosus muscle clearly seen before the lesion (Fig. 10A, black arrow) disappeared on the left after hemisection and did not recover after $42 \mathrm{~d}$ of training. Second, the first burst in EDB muscles seen at mid-cycle before the lesion (Fig. 10B, black arrow) was severely reduced on the left after the hemisection and training. Third, the amplitude of VL bursts on both sides was similar before the lesion (Fig. 10C, blue).
However, there was a clear reduction of the overall EMG envelope on the left and an increase on the right after hemisection and training. The increase in the right knee extensor may help in bearing some weight shift on the right side. Fourth, the overall amplitude of EMG activity of ankle extensors GM appeared to be increased on both sides after the hemisection and training. The crouched posture at the ankles (see, e.g., Fig. 2) might increase stretch-related excitatory sensory feedback to GM motor pools.

We attempted to correlate the size of the lesions of the LTMtrained cats (Fig. 7) with either the individual evolution of cats throughout the recovery period or their performance at $42 \mathrm{~d}$ after lesion (Fig. 11), the last day of recordings. As with most spinal lesion studies, a variety of mechanisms (spinal, propriospinal, and supraspinal) take part in the recovery, and it is close to impossible to attribute a given defect or recovery to a very specific tract. Furthermore, cats may adopt a strategy that is individually more comfortable. Nevertheless, considering our limited sample, it is quite clear that Cats 1 and 5 showed more locomotor deficits. Both had very large lesions (especially Cat 5). Cat 1 initially walked on the rungs for only a few steps (Fig. 11, histogram, gray bars) and then started to slip on the rungs and touch ground between rungs (orange). Finally, for all subsequent days, the cat walked regularly with the LH between the rungs (blue) and all other limbs on the rungs. Cat 5 walked often between rungs (histograms, blue bars) but also had more sequences slipping on the rungs (orange bars). On the other hand, Cats 2, 3, 6, and 7 clearly 


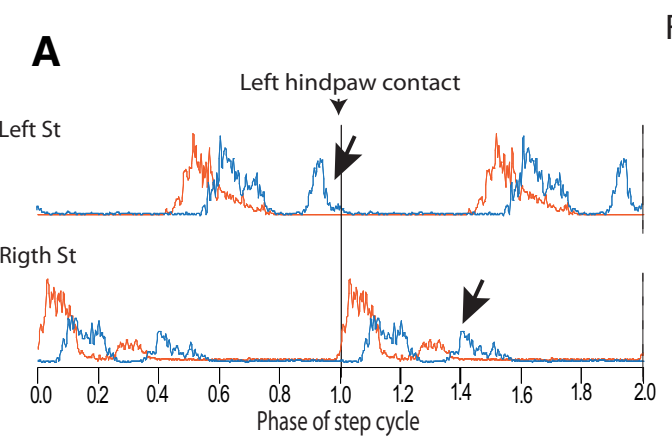

Flexors
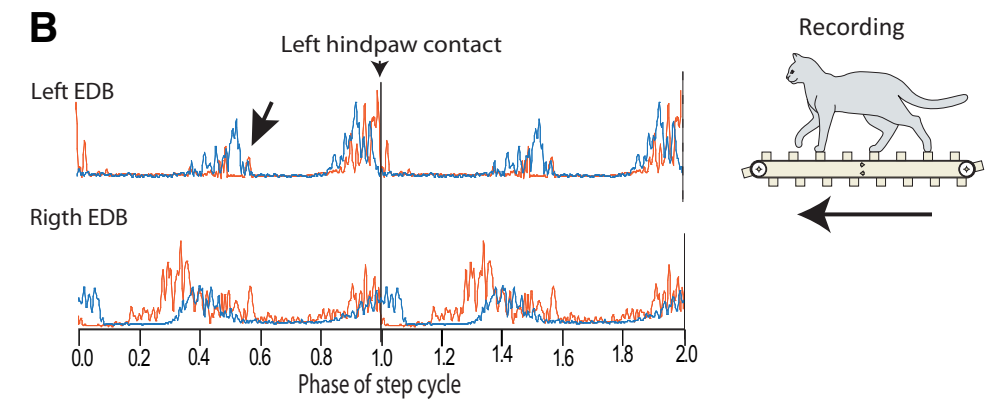

Extensors $\quad$ LTM 42 days after lesion
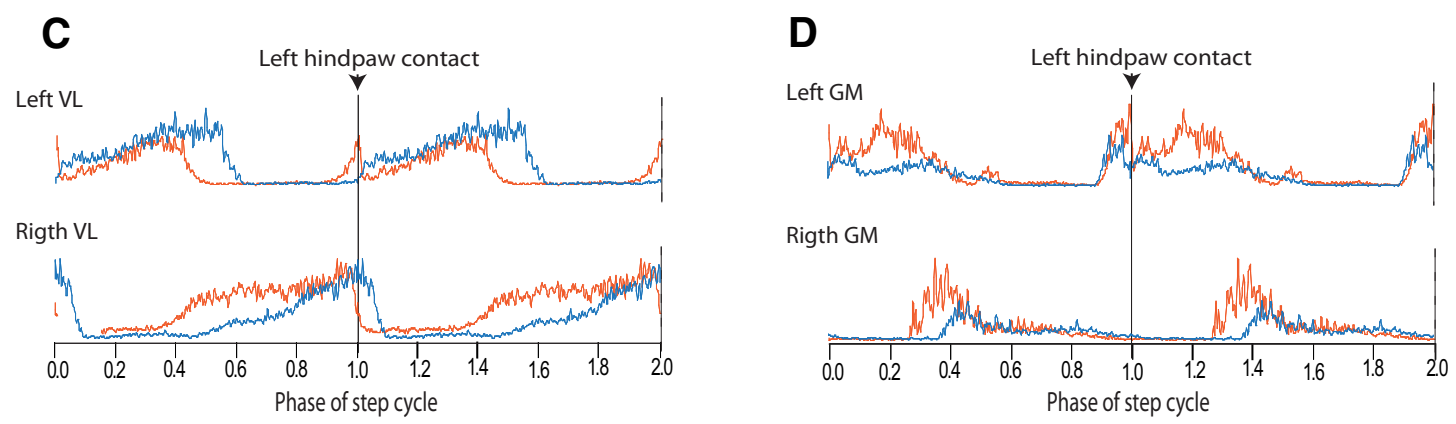

Figure 10. Averaged rectified EMG locomotor bursts synchronized on the left paw contact in Cat $1(\boldsymbol{A}, \boldsymbol{C}, \boldsymbol{D})$ and Cat $3(\boldsymbol{B})$ comparing stepping on the LTM before (blue lines) and $42 \mathrm{~d}$ after a left lateral hemisection (orange lines). Black arrows indicate three major changes: (1) the second burst of activity in the semitendinosus muscle on the left disappears after the hemisection; (2) the second burst of activity on the right is still present after the hemisection; and (3) an important decrease (or disappearance in some cats) of the EDB's first burst on the side of the lesion. St, Semitendinosus.

evolved toward a pattern of walking on the rungs (gray bars) with all 4 limbs at $42 \mathrm{~d}$ after hemisection. Cat 6 with the smallest lesion succeeded to step mainly on the rungs the earliest, at $28 \mathrm{~d}$.

\section{Walking on the FTM after FTM or LTM training}

Whereas the previous section compared the various patterns of locomotor adaptations when walking on the LTM and FTM, the present section illustrates walking characteristics of hemisected cats walking on the FTM after having been trained on the LTM or the FTM for $42 \mathrm{~d}$. It is important to note that the cats here are assessed on the FTM after they have been trained either on the LTM or the FTM, so that this is the "outcome" of the training in both conditions.

\section{Interlimb coupling after hemisection in LTM- and FTM-trained groups}

Figure 12 details changes in interlimb coupling of cats walking on the FTM after being trained either on the FTM or the LTM. Following both types of training, diagonal couplings were rarely found after hemisection; therefore, lateral coupling prevailed on the FTM with an asymmetry between left and right lateral coupling as illustrated on the right side of the panel for LTM- and FTM-trained groups. Quite clearly, cats with a left hemisection preferred a gait with a predominance of right lateral coupling (i.e., coupling of limbs contralateral to the lesion independently of the training regimen). As was shown previously in Figure $4 B$ (left, right, horizontal bars), although there was a predominance, in intact cats, of diagonal over lateral couplings, both types of coupling were symmetrical, whereas here the lateral couplings after hemisection were clearly asymmetrical whether cats were trained on the LTM or FTM. In other words, the asymmetry in lateral coupling between forelimbs and hindlimbs seen after the hemisection was not improved by either form of training.

\section{Limb kinematics and angular excursion}

Figure 13 shows limb kinematics of the LH of Cat 3 while walking on the FTM before hemisection (blue traces) and after $42 \mathrm{~d}$ of LTM training (red traces). Figure 13A (top, figurines) indicates the conditions of recording and training. There was an increase in the knee and ankle angles of the hemisected cat walking on the FTM after being trained on the LTM compared with the same cat with an intact spinal cord. These findings were consistent among LTM-trained cats. In some cats, the ankle joint showed a greater yield (Fig. 13B, black arrow) during the whole stance phase.

Measurements were made of cycle durations on the FTM at various time intervals $(7,28,42 \mathrm{~d})$ during the locomotor training on FTM or LTM. For the LTM-trained group, the swing phases measured on the FTM were statistically longer on both sides with clear reciprocal compensatory changes of the stance duration on both sides leading to an unchanged cycle duration overall on both sides.

\section{Trajectory of the toes}

Figure 14 shows the trajectory of the toes during the swing phase of stepping on the FTM of one hemisected cat trained on the LTM (Cat 1) and another hemisected (Cat 8) trained on the FTM. For each cat, we superimposed the toe trajectories when the cat had an intact spinal cord with that after $42 \mathrm{~d}$ of either form of training.

With FTM training (Fig. 14, right, Cat 8), the toes followed a trajectory with a very similar shape but with a much larger amplitude (compare blue and orange traces). This was found in all cats trained on the FTM after hemisection. With LTM training 


\section{CAT 1}

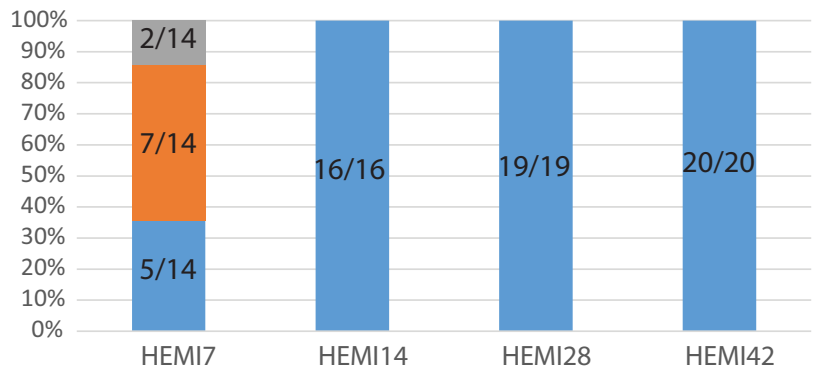

CAT 3

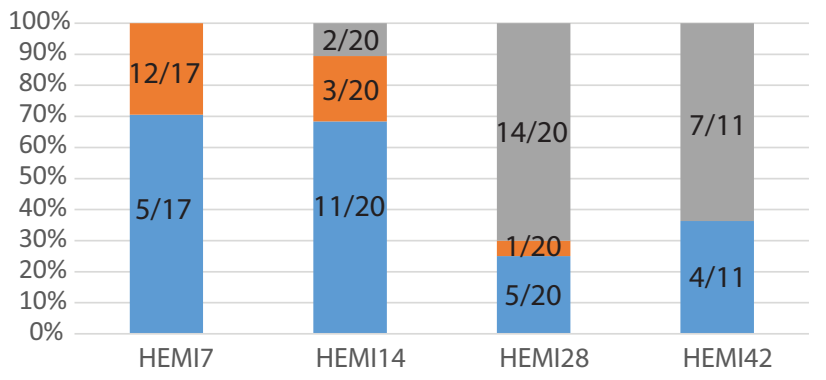

CAT 6

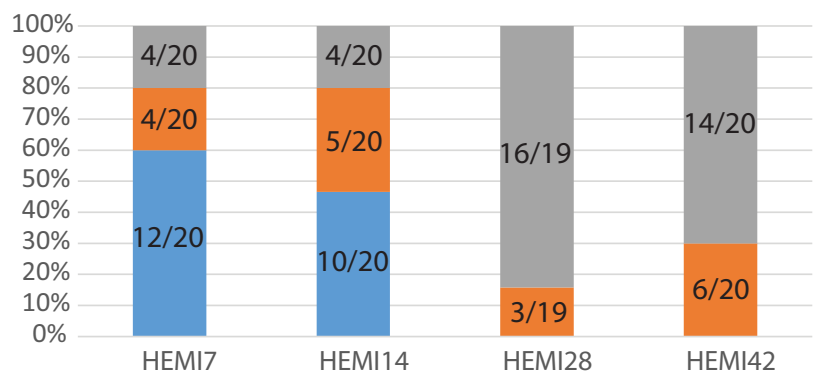

CAT 2

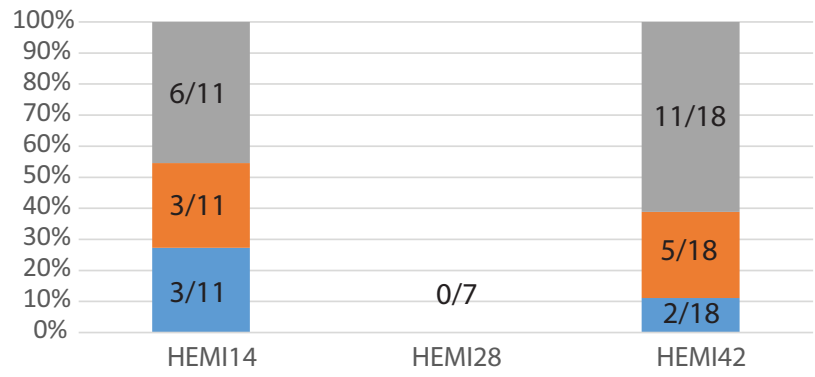

CAT 5

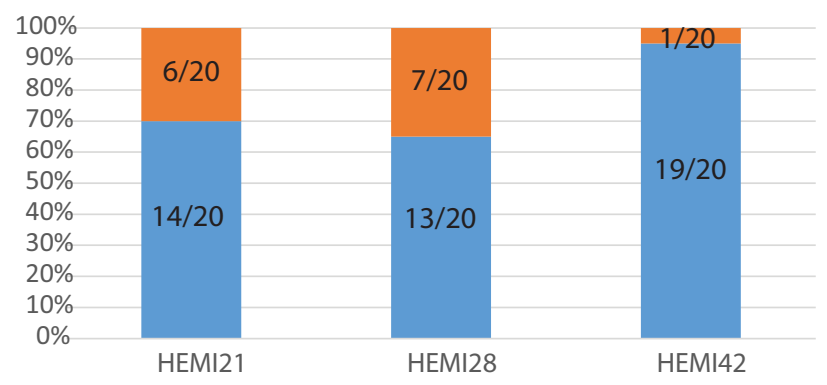

CAT 7

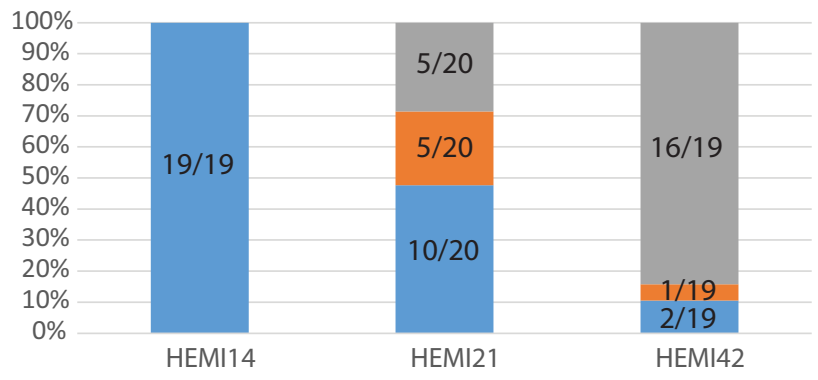

\section{On rungs}

Full slide

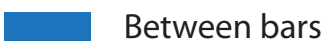

Figure 11. Paw placement patterns after spinal cord injury during LTM stepping. Three forms of left hindpaw placement were observed during LTM training sessions: (1) on the rungs ( $g$ ray bars), where the left affected paw was placed on the rung; (2) full slide (orange bars), the left paw contacted the rung, but it glided to the bottom of the space between rungs); and (3) between bars (blue bars), the left hindpaw was placed directly between two consecutives rungs. Cats recover trough time paw placement on the rungs, except for Cats 1 and 5 , which kept systematically placing the paw between rungs.

(Fig. 14, left panels, Cat 1), interestingly, the trajectory (orange trace) was completely different. Before the lesion (blue trace), the shape was similar as previously described for cats trained on FTM (i.e., similar to the shape of the blue trace on the right panel). After training on the LTM, the toes elevated early in swing (from right to left), but the trajectory continued upward lift to the end of the swing phase where it finally dropped abruptly to contact with the treadmill surface. This particular trajectory already appeared $7 \mathrm{~d}$ after the lesion and maintained throughout the whole training period. This unusual trajectory was seen in 4 of the 6 cats trained on the LTM at the end of the training protocol.

Of great interest is that, in both LTM and FTM-trained cats, the "hook" representing the terminal foot placement observed before the lesion (Fig. 14, black arrows) disappeared. Instead, the toes adopted a brisk vertical trajectory to contact the surface of the treadmill. This abrupt landing appears to result from the hemisection and was not corrected by training.

We also compared the foot drag of 2 cats with similar spinal damage (Cat 8 trained on the FTM and Cat 1 on the LTM; Fig. 7). After $28 \mathrm{~d}$, there was a reduction of the percentage of steps with foot drag with both types of training. After $42 \mathrm{~d}$, there were no more steps with foot drag in the LTM-trained cat, whereas 30\% of steps showed foot drag in the FTM-trained cat.

\section{EMG activity during FTM stepping}

Although there were clear adaptations in EMG activity in some muscles when walking on the LTM before and after the hemisection, when walking on the FTM (the outcome), the EMG profiles 
LTM trained group

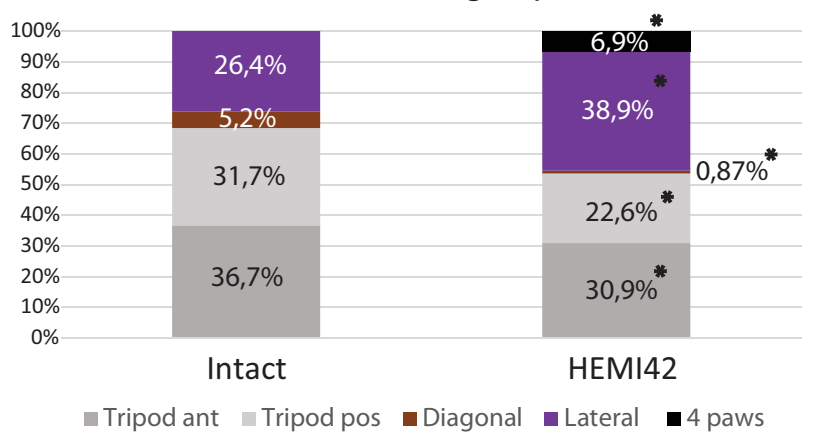

FTM trained group

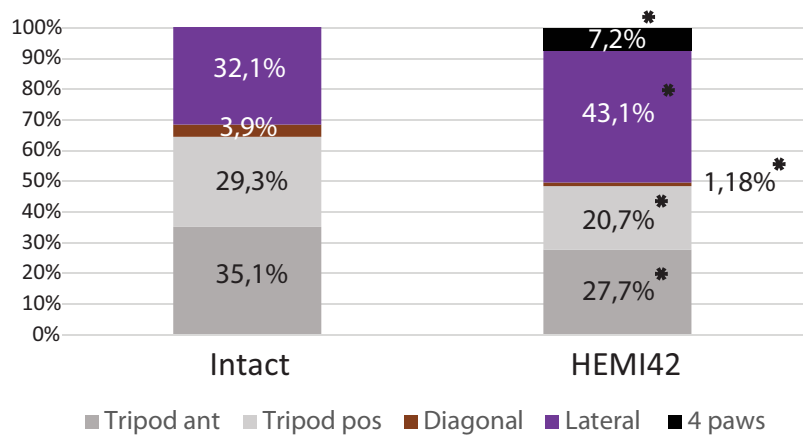

Diagonal/lateral couplets LTM trained group 42 days after hemisection

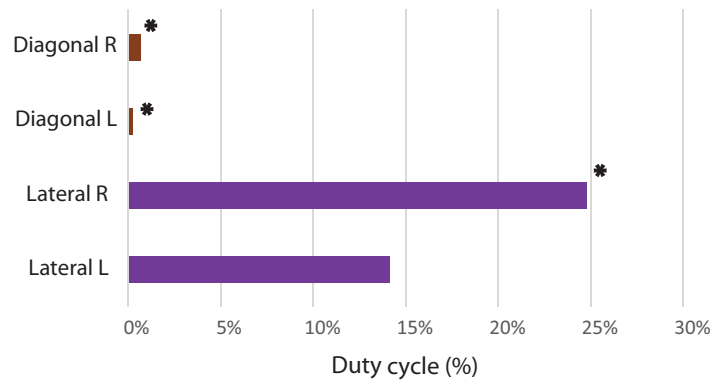

Diagonal/lateral couplets FTM trained group 42 days after hemisection

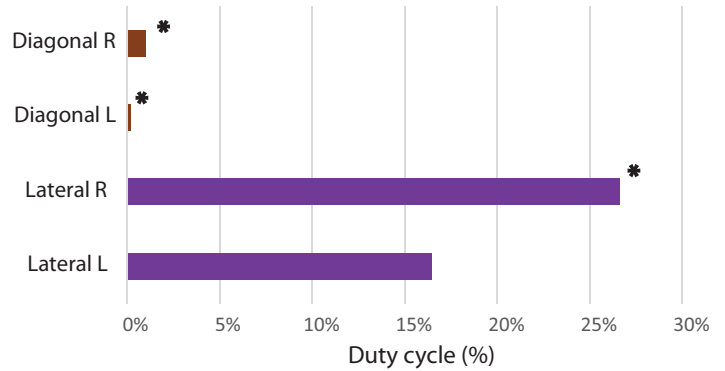

Figure 12. Footfall patterns illustrate the percentage of the duty cycle with different feet on the ground comparing FTM- and LTM-trained groups before and $42 \mathrm{~d}$ after spinal hemisection during FTM stepping. Values within each portion of bars indicate the amount of time the cat spent in a particular combination of limbs in contact with the ground. Gray rectangles represent periods where three feet are on the ground: either two hindlimbs and one forelimb (tripod posterior) or two forelimbs and one 1 hindlimb (tripod anterior). Violet rectangles represent periods in violet in $\boldsymbol{A}$ where the two limbs on one side are coupled (lateral coupling). Brown rectangles represent diagonal coupling. The distribution of interlimb coupling is pooled for left and right coupling. Bottom for both FTM and LTM, details of the type of coupling: diagonal right or left and lateral right or left. L, Left; R, right. ${ }^{*} p<0.05$, within the same condition (FTM- or LTM-trained groups).

of LTM-trained cats did not differ from FTM-trained cats. Apparently, adaptations in EMG activity developed to step on rungs were not transferred to normal stepping on an FTM.

\section{Discussion}

\section{The LTM}

Locomotor abilities of cats after large CNS lesions, such as a decerebration or spinal sections, are often assessed on an FTM or on a walkway. Although cats can walk even with severe CNS lesions on an FTM, their ability to perform precise foot placement is often overlooked, although such precision walking is essential in daily life. The main ideas of the present study are as follows: (1) to develop a treadmill to assess locomotion on the rungs of a moving LTM requiring precise voluntary foot positioning; (2) to assess the capability of cats after a spinal hemisection to cope with such a demanding locomotor skill; and (3) to regularly train animals in such a demanding task with the underlying hypothesis that training with this added difficulty could improve locomotor recovery even on an FTM after lesions because it maximizes not only changes in spinal locomotor circuits but also remnant supraspinal inputs.

The advantage of LTM compared with a horizontal fixed ladder is that it allows to maintain the same environment as for walking on an FTM with the added constraint of walking precisely on moving rungs. Thus, visual inputs of the moving rungs constitute an undisputable supraspinal necessary control to perform voluntary locomotion on the rungs or between the rungs of an LTM. Finally, confinement to a treadmill enclosure while walking on the LTM permitted to average locomotor parameters (EMG and kinematics) of several cycles in the same stationary position relative to the frame of reference as is the case with FTM. Changes of kinematics or EMGs may then be attributed to modifications of the tasks in one environment and not to two different tasks in two different environments.

Other studies have investigated the ability of cats to walk in more challenging conditions (Smith et al., 1998). Such is the case for walking on a horizontal ladder or between barriers in a walkway (Beloozerova and Sirota, 1993; Beloozerova et al., 2010) or a split-belt treadmill (D’Angelo et al., 2014). Work by Krouchev and Drew (2013) has focused on gait modifications when negotiating an obstacle on a treadmill. Although these studies contributed considerably to our understanding of the locomotor system capabilities, they were not as such involved in evaluating the locomotor performance after CNS lesions or the effects of training.

Approaches requiring step-by-step adjustments on visual targets projected on a treadmill (C-Mill) are currently provided for human locomotor training after stroke. This is quite a different task and requires different constraints (Heeren et al., 2013; Hollands et al., 2013). Such a paradigm would be unrealistic for the questions asked here, especially for animal experiments in which precise foot targeting is desired.

\section{Kinematic and EMG changes between FTM and LTM}

Our walking paradigm requires a precise foot placement at every step and consequently involves supraspinal inputs. The interrung spacing was adjusted for each cat so that the locomotor 


\section{CAT 3}
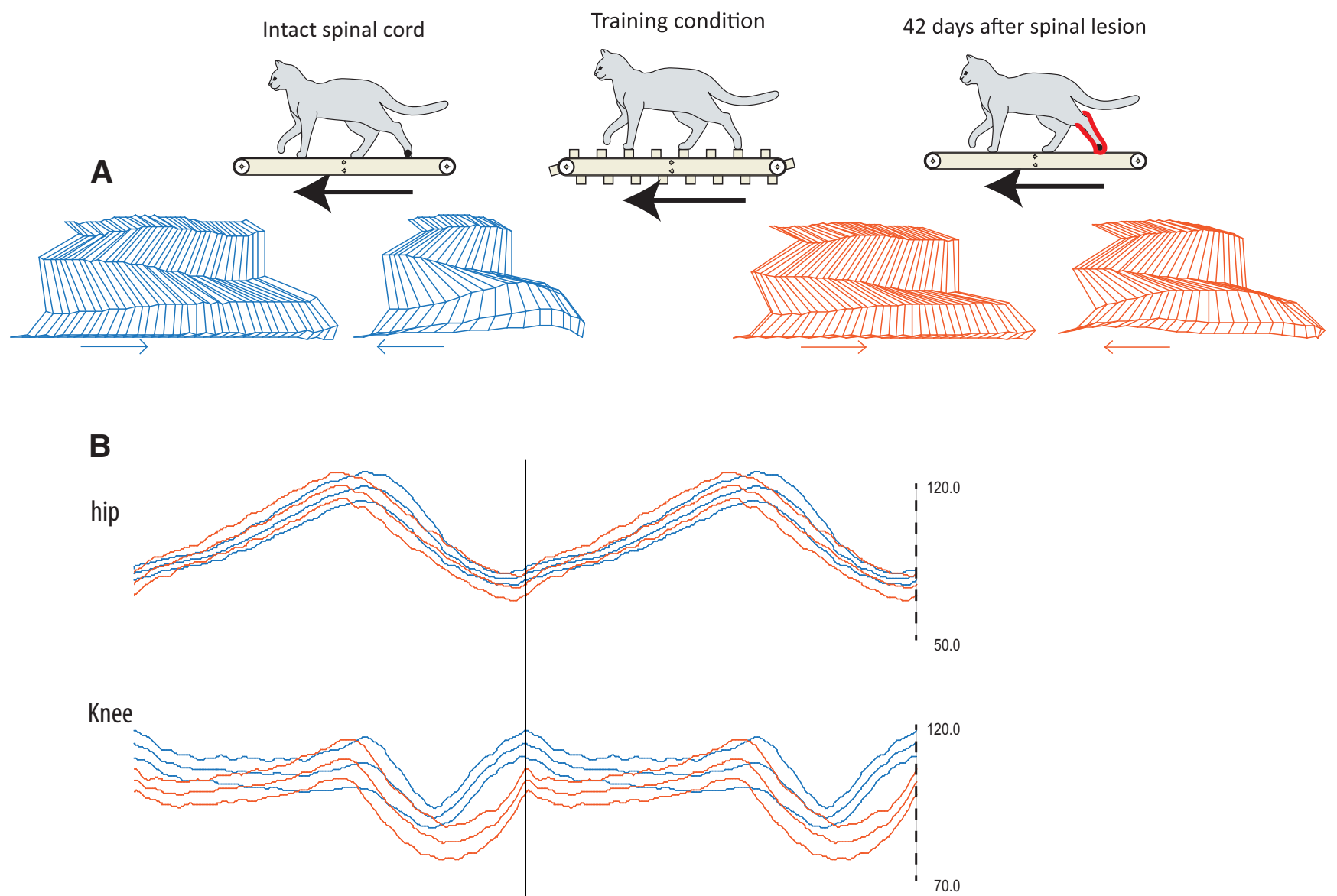

ankle

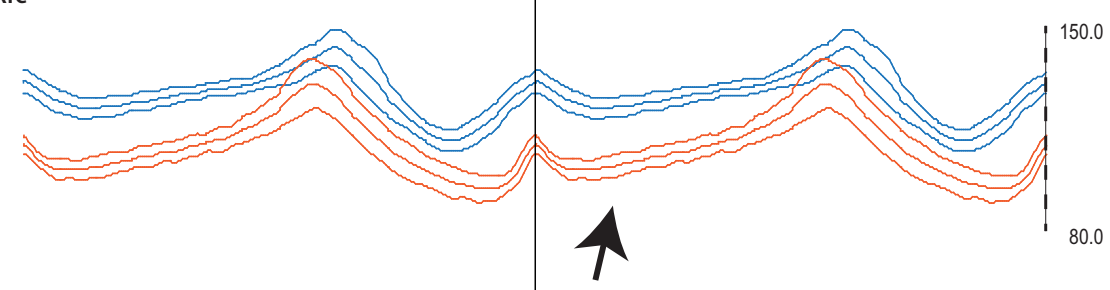

MTP

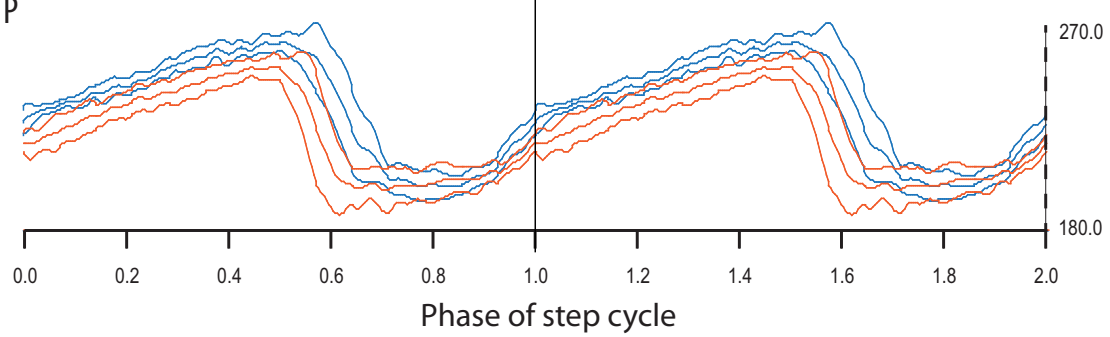

Intact spinal cord

42 days after spinal lesion

Figure 13. A, Stick figures of swing and stance phase from one step cycle of the LH of (at 3, during FTM stepping $42 \mathrm{~d}$ after spinal hemisection and trained on the LTM (orange lines) compared with the same kinematic parameters of the same cat before spinal lesion (blue lines). $\boldsymbol{B}$, Averaged angular excursion of the hip, knee, ankle, and MTP joints of the LH synchronized on the left paw contact for the same cat on the FTM before and 6 weeks after training on the LTM.

characteristics of the step cycles were similar for both FTM and LTM. This provided a comfortable stride length for each cat as described previously (Beloozerova and Sirota, 1993; Beloozerova et al., 2010). It also allowed attributing changes in locomotor characteristics (angular excursion, EMGs) to the task rather than to the overall kinematic requirements (e.g., step cycle adjustments). Despite a significant change in difficulty, the kinematic changes in LTM walking were modest as seen in Figure 2. The interlimb coordination favored a stable diagonal interlimb coupling (Fig. 4). The trajectory of the foot of the LH tended to reach 


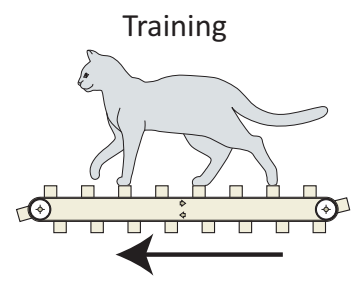

CAT 1

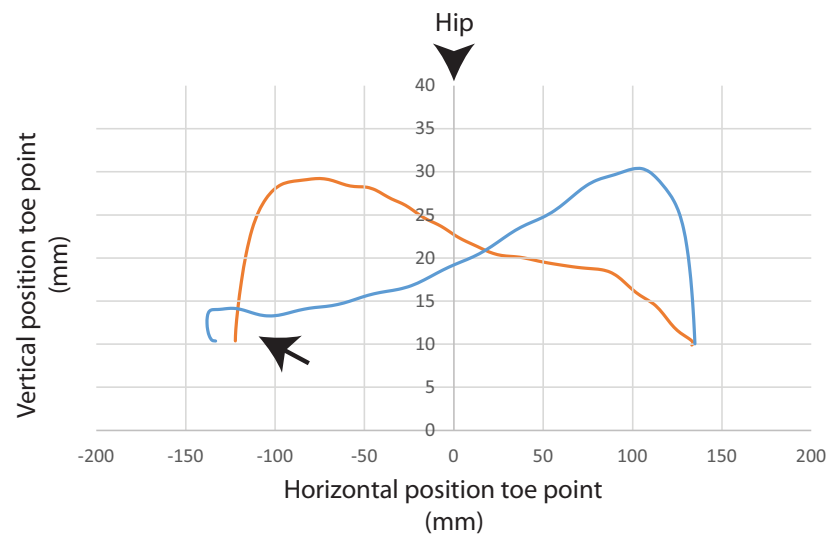

Training

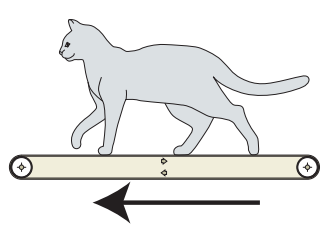

CAT 8

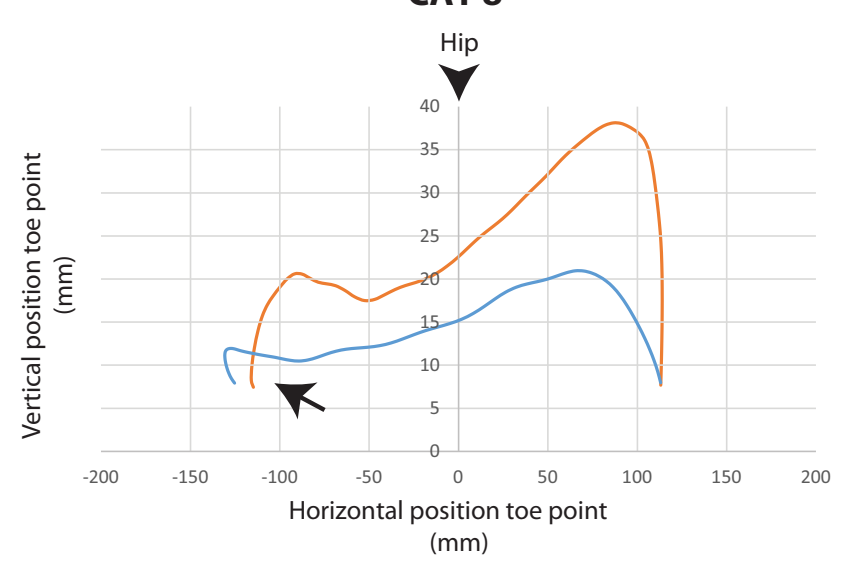

Recording

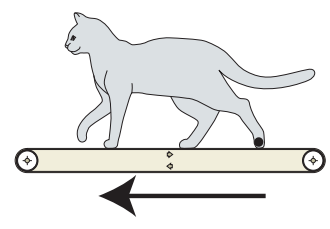

- Intact spinal cord

42 days after spinal lesion

Figure 14. Graphical 2D representations of left toe trajectories during FTM stepping before (blue lines) and after (orange lines) spinal hemisection. Left, Cat 1 trained on the LTM. Right, Cat 8 trained on the FTM. A "hook" shape was seen before spinal hemisection as the foot prepared to land, whereas this hook disappears (black arrow) after hemisection. Cat figurines represent training conditions for each panel. Arrows below figurines indicate the direction of stepping.

an increased height value in mid-swing in some cats, whereas, in others, the height did not significantly change (Fig. 3). We found that, just before touch down, the toes made a slight upward movement before extending to contact the ground or the rung, which was reflected as a "hook" in the toe trajectory (as seen in all cats, Fig. 3). This precise positioning is important (Halbertsma, 1983) and did not apparently change between the two tasks.

Specific changes in EMGs may subserve some kinematic adaptations. Of note are the increased amplitude and duration of the first burst of semitendinosus (Fig. 6), which is associated with the onset of the swing phase. The increase in amplitude of the second burst of semitendinosus associated with foot landing was clearly more important during LTM walking. We postulate that this second burst may serve to control the end of the swing and allow the foot to be placed on the rungs. This second semitendinosus burst coincides with a period of intense discharge in knee flexor proprioceptive afferents (Prochazka et al., 1976). This period when knee flexors are stretched before landing corresponds to an increase in reflex excitability and could lead to an increase of this second EMG burst. Such a mechanism was suggested for humans, also (Duysens et al., 1998). However, the disappearance of this second semitendinosus burst after hemisection (Fig. 10A) rather suggests that the muscle is used as part of a strategy of central origin.

\section{Locomotor capabilities after hemisection}

Following a unilateral spinal cord hemisection, the limb caudal to the hemisection is at first flaccid but can recover extensive locomotor capabilities after 3-8 weeks (Jane et al., 1964) and older (Eidelberg, 1983). Such recovery also appears after a staggered hemisection at two spinal levels (Kato et al., 1984) performed to investigate compensatory mechanisms from contralateral descending intact fibers. In this case, cats recovered complex motor behavior, such as jumping on and off a $25 \mathrm{~cm}$ ledge. Others also found a recovery of function for several months after the hemisection as well anatomical sprouting of afferents on the side of the hemisection (Murray and Goldberger, 1974; Helgren and Goldberger, 1993) suggesting these might play a role in recovery. Our own previous studies on hemisected cats clearly documented the recovery of locomotion. (Martinez et al., 2013).

In the present work, we show that hemisected cats can use individual strategies when walking on the LTM even in cats with similar lesions. Some cats placed the foot between rungs while the three other feet were placed on the rungs. This led to a limping walk, which apparently was more stable for some cats. This seems to be a voluntary strategy, not a random placement strategy, because the hind foot was put correctly and repetitively between the rungs. Work using cortical recordings during walking between widely spaced barriers has shown that the motor cortex is very active (Beloozerova and Sirota, 1993), which suggests a major 
participation in these complex strategies. This situation, however, is more akin to sequentially stepping over individual obstacles, whereas our task requires a step-by-step correction. In our study, cats evolved to walk on the rungs or between the rungs, but at the end of training, cats did not switch between strategies (i.e., to walk on or between the rungs) but adopted a stable strategy, between or on the rungs (as seen in Figs. 8, 11). As discussed in Results, establishing a correlation between the size of the lesions and the ability to walk on the rungs $42 \mathrm{~d}$ after lesion is somewhat problematic because it would require a greater number of animals and a study over a longer time period for the recovery. Six weeks is a relatively short recovery period for large spinal lesions in cats performing a complex task. This LTM paradigm would be very appropriate to perform such longer-term studies.

\section{Effects of FTM/LTM training on FTM walking}

One of the questions addressed in this work is whether locomotor training can durably change locomotor characteristics when the spinal cord is either intact or hemisected. This is a fundamental issue because the basis of rehabilitation therapies may be to change characteristics of basic motor patterns, such as locomotion.

When assessing locomotion on the FTM (the "outcome") in cats trained on the FTM or the LTM after hemisection, there were no dramatic changes in overall kinematic parameters, but more detailed analysis showed that the foot trajectory can be changed importantly in some cats (Fig. 3). Figure 14 shows 2 cats trained for $42 \mathrm{~d}$ either on the FTM or the LTM after a hemisection. When trained on the FTM, the paw trajectory keeps the same overall shape but is much increased in amplitude (Cat 8). When trained on the LTM, Cat 1 displayed a trajectory with the toes elevating toward the end of the swing as if to possibly reach a forward target (e.g., a rung). Therefore, training and training regimen could affect some locomotor characteristics on long term.

Most remarkable, however, was that the hook previously described and representing a soft landing on the rung disappeared in the LH after hemisection. Figure 14 compares foot landing with intact spinal cord and $42 \mathrm{~d}$ after spinal hemisection. As can be seen, the foot now drops straight to the contact surface (Fig. 14 , red/orange trace). This probably corresponds to the abolition of the second burst in semitendinosus muscle, which we associated before with foot landing in the intact (on the FTM or LTM). Neither FTM nor LTM training reestablished the second semitendinosus burst or the precontact hook.

Finally, we found that foot drag often seen in hemilesioned cats was much improved in cats trained on the LTM compared with training on the FTM. Both the number of steps with foot drag diminished but also the percentage of the swing phase occupied by foot drag.

In conclusion, this work is the first step toward the development of a method to evaluate the voluntary component of locomotion required for precision walking. We also propose this method to train regularly cats (or rodents) on an LTM, which requires the involvement not only of spinal locomotor circuits but also of some supraspinal structures. Our preliminary evidence points to the possibility of inducing durable changes in the locomotor characteristics similar to spinal task learning (Hodgson et al., 1994). Training in demanding conditions, such as LTM walking after CNS lesions, could potentially improve the locomotor performance of animals in less demanding conditions after CNS lesions.

\section{References}

Alluin O, Delivet-Mongrain H, Gauthier MK, Fehlings MG, Rossignol S, Karimi-Abdolrezaee S (2014) Examination of the combined effects of chondroitinase $\mathrm{ABC}$, growth factors and locomotor training following compressive spinal cord injury on neuroanatomical plasticity and kinematics. PLoS One 9:e111072. CrossRef Medline

Amos A, Armstrong DM, Marple-Horvat DE (1987) A ladder paradigm for studying skilled and adaptive locomotion in the cat. J Neurosci Methods 20:323-340. CrossRef Medline

Armstrong DM, Marple-Horvat DE (1996) Role of the cerebellum and motor cortex in the regulation of visually controlled locomotion. Can J Physiol Pharmacol 74:443-455. CrossRef Medline

Barbeau H, Rossignol S (1987) Recovery of locomotion after chronic spinalization in the adult cat. Brain Res 412:84-95. CrossRef Medline

Barbeau H, Ladouceur M, Mirbagheri MM, Kearney RE (2002) The effect of locomotor training combined with functional electrical stimulation in chronic spinal cord injured subjects: walking and reflex studies. Brain Res Brain Res Rev 40:274-291. CrossRef Medline

Barrière G, Leblond H, Provencher J, Rossignol S (2008) Prominent role of the spinal central pattern generator in the recovery of locomotion after partial spinal cord injuries. J Neurosci 28:3976-3987. CrossRef Medline

Barrière G, Frigon A, Leblond H, Provencher J, Rossignol S (2010) Dual spinal lesion paradigm in the cat: evolution of the kinematic locomotor pattern. J Neurophysiol 104:1119-1133. CrossRef Medline

Beloozerova IN, Sirota MG (1993) The role of the motor cortex in the control of accuracy of locomotor movements in the cat. J Physiol 461:1-25. CrossRef Medline

Beloozerova IN, Farrell BJ, Sirota MG, Prilutsky BI (2010) Differences in movement mechanics, electromyographic, and motor cortex activity between accurate and nonaccurate stepping. J Neurophysiol 103:22852300. CrossRef Medline

D’Angelo G, Thibaudier Y, Telonio A, Hurteau MF, Kuczynski V, Dambreville C, Frigon A (2014) Modulation of phase durations, phase variations and temporal coordination of the four limbs during quadrupedal split-belt locomotion in intact adult cats. J Neurophysiol 112:1825-1837. CrossRef Medline

De Leon RD, Hodgson JA, Roy RR, Edgerton VR (1998) Full weight-bearing hindlimb standing following stand training in the adult spinal cat. J Neurophysiol 80:83-91. Medline

De Leon RD, Hodgson JA, Roy RR, Edgerton VR (1999) Retention of hindlimb stepping ability in adult spinal cats after the cessation of step training. J Neurophysiol 81:85-94. Medline

Dietz V (1995) Locomotor training in paraplegic patients. Ann Neurol 38: 965. CrossRef Medline

Drew T, Kalaska J, Krouchev N (2008) Muscle synergies during locomotion in the cat: a model for motor cortex control. J Physiol 586:1239-1245. CrossRef Medline

Duysens J, van Wezel BM, van de Crommert HW, Faist M, Kooloos JG (1998) The role of afferent feedback in the control of hamstrings activity during human gait. Eur J Morphol 36:293-299. CrossRef Medline

Edgerton VR, De Leon RD, Tillakaratne N, Recktenwald MR, Hodgson JA, Roy RR (1997) Use-dependent plasticity in spinal stepping and standing. Adv Neurol 72:233-247. Medline

Edgerton VR, Leon RD, Harkema SJ, Hodgson JA, London N, Reinkensmeyer DJ, Roy RR, Talmadge RJ, Tillakaratne NJ, Timoszyk W, Tobin A (2001) Retraining the injured spinal cord. J Physiol 533:15-22. CrossRef Medline

Eidelberg E (1983) Loss and recovery of locomotor function after spinal cord lesions in cats and monkeys. In: Nerve organ and tissue regeneration: research perspectives (Seil FJ, ed), pp 231-242. San Diego: Academic.

Engberg I, Lundberg A (1969) An electromyographic analysis of muscular activity in the hindlimb of the cat during unrestrained locomotion. Acta Physiol Scand 75:614-630. CrossRef Medline

Farrell BJ, Bulgakova MA, Sirota MG, Prilutsky BI, Beloozerova IN (2015) Accurate stepping on a narrow path: mechanics, EMG, and motor cortex activity in the cat. J Neurophysiol 114:2682-2702. Medline

Field-Fote EC, Lindley SD, Sherman AL (2005) Locomotor training approaches for individuals with spinal cord injury: a preliminary report of walking-related outcomes. J Neurol Phys Ther 29:127-137. CrossRef Medline

Gorassini MA, Prochazka A, Hiebert GW, Gauthier MJ (1994) Corrective responses to loss of ground support during walking: I. Intact cats. J Neurophysiol 71:603-610. Medline

Halbertsma JM (1983) The stride cycle of the cat: the modelling of locomotion by computerized analysis of automatic recordings. Acta Physiol Scand Suppl 521:1-75. Medline 
Harkema SJ (2001) Neural plasticity after human spinal cord injury: application of locomotor training to the rehabilitation of walking. Neuroscientist 7:455-468. CrossRef Medline

Harkema SJ, Behrman AL, Barbeau H (2011) Locomotor training: principles and practice. New York: Oxford UP.

Heeren A, van Ooijen M, Geurts AC, Day BL, Janssen TW, Beek PJ, Roerdink M, Weerdesteyn V (2013) Step by step: a proof of concept study of C-Mill gait adaptability training in the chronic phase after stroke. J Rehabil Med 45:616-622. CrossRef Medline

Helgren ME, Goldberger ME (1993) The recovery of postural reflexes and locomotion following low thoracic hemisection in adult cats involves compensation by undamaged primary afferent pathways. Exp Neurol 123:17-34. CrossRef Medline

Hildebrand M (1976) Analysis of tetrapod gaits: general considerations and symmetrical gaits. In: Neural control of locomotion (Herman RM, Grillner S, Stein PS, Stuart DG, eds), pp 203-236. New York: Plenum.

Hodgson JA, Roy RR, De Leon R, Dobkin B, Edgerton VR (1994) Can the mammalian lumbar spinal cord learn a motor task? Med Sci Sports Exerc 26:1491-1497. Medline

Hollands KL, Pelton T, Wimperis A, Whitham D, Jowett S, Sackley C, Alan W, van Vliet P (2013) Visual cue training to improve walking and turning after stroke: a study protocol for a multi-centre, single blind randomised pilot trial. Trials 14:276. CrossRef Medline

Jane JA, Evans JP, Fisher LE (1964) An investigation concerning the restitution of motor function following injury to the spinal cord. J Neurosurg 21:167-171. CrossRef Medline

Kato M, Murakami S, Yasuda K, Hirayama H (1984) Disruption of foreand hindlimb coordination during overground locomotion in cats with bilateral serial hemisection of the spinal cord. Neurosci Res 2:27-47. CrossRef Medline

Krouchev N, Drew T (2013) Motor cortical regulation of sparse synergies provides a framework for the flexible control of precision walking. Front Comput Neurosci 7:83. CrossRef Medline

Lavoie S, McFadyen B, Drew T (1995) A kinematic and kinetic analysis of locomotion during voluntary gait modification in the cat. Exp Brain Res 106:39-56. Medline

Martinez M, Delivet-Mongrain H, Leblond H, Rossignol S (2011) Recovery of hindlimb locomotion after incomplete spinal cord injury in the cat involves spontaneous compensatory changes within the spinal locomotor circuitry. J Neurophysiol 106:1969-1984. CrossRef Medline

Martinez M, Delivet-Mongrain H, Rossignol S (2013) Treadmill training promotes spinal changes leading to locomotor recovery after partial spinal cord injury in cats. J Neurophysiol 109:2909-2922. CrossRef Medline

Murray M, Goldberger ME (1974) Restitution of function and collateral sprouting in the cat spinal cord: the partially hemisected animal. J Comp Neurol 158:19-36. CrossRef Medline

Patla AE, Prentice SD, Robinson C, Neufeld J (1991) Visual control of locomotion: strategies for changing direction and for going over obstacles. J Exp Psychol Hum Percept Perform 17:603-634. CrossRef Medline

Pearson KG, Rossignol S (1991) Fictive motor patterns in chronic spinal cats. J Neurophysiol 66:1874-1887. Medline

Prochazka A, Westerman RA, Ziccone SP (1976) Discharges of single hindlimb afferents in the freely moving cat. J Neurophysiol 39:1090-1104. Medline

Rossignol S, Martinez M, Escalona M, Kundu A, Delivet-Mongrain H, Alluin O, Gossard JP (2015) The "beneficial" effects of locomotor training after various types of spinal lesions in cats and rats. In: Sensorimotor rehabilitation: at the crossroad of basic and clinical sciences (Dancause N, Nadeau S, Rossignol S, eds), pp 173-198. Oxford: Elsevier.

Smith AC, Mummidisetty CK, Rymer WZ, Knikou M (2014) Locomotor training alters the behavior of flexor reflexes during walking in human spinal cord injury. J Neurophysiol 112:2164-2175. CrossRef Medline

Smith JL, Chung SH, Zernicke RF (1993) Gait-related motor pattern and hindlimb kinetics for the cat trot and gallop. Exp Brain Res 94:308-322. Medline

Smith JL, Carlson-Kuhta P, Trank TV (1998) Forms of forward quadrupedal locomotion: III. A comparison of posture, hindlimb kinematics, and motor patterns for downslope and level walking. J Neurophysiol 79:1702_ 1716. Medline 